\title{
AN OVERVIEW OF ENERGY PRODUCTION FROM ANIMAL WASTE DURING IRAN'S ENERGY TRANSITION: IMPLICATION OF MANURE CHEMICAL COMPOSITION
}

\author{
DARYABEIGI ZAND, A. ${ }^{{ }^{*}}-$ RABIEE ABYANEH, M. ${ }^{1}-$ KHODAEI, H. R. ${ }^{2}$ \\ ${ }^{I}$ School of Environment, College of Engineering, University of Tehran, Tehran, Iran \\ ${ }^{2}$ Department of Animal Science, Islamic Azad University, Golpayegan Branch, Isfahan, Iran \\ *Corresponding author \\ e-mail:adzand@ut.ac.ir \\ (Received $15^{\text {th }}$ Jun 2018; accepted $7^{\text {th }}$ Aug 2018)
}

\begin{abstract}
Biogas is a renewable energy source that is generated by the decomposition of organic waste in anaerobic process. The theoretical biogas potential is defined as the possible amount of gas production from biomass. This potential can be evaluated using various calculation methods each of which considers different parameters. The objective of this study was therefore to compare the potential of biogas energy generation from the livestock waste produced from the animal husbandry in Iran using different calculation methods. Using 4 methods of calculation indicated that the amount of 11.82, 5.25, 16.05 and 2.1 million ton of animal waste could be produced in Iran in 2016 with a biogas generation potential of $886.57,173.36,745.37$ and 565.85 million $\mathrm{m}^{3}$, energy equivalent of 5.14, 1, 4.32 and 3.28 thousand $\mathrm{GWh}$ and electricity generation of $1.59,0.31,1.34$ and 1.02 thousand $\mathrm{GWh}$. These amounts of electricity generation could provide $2.03 \%, 1.71 \%, 0.39 \%$ and $1.3 \%$ of the electrical energy consumed in household sector in 2016 in Iran, respectively. Furthermore the methane yield potential from the livestock manure with considering the different types of substrate (lipid, lignin, dry matter and cellulose) was evaluated. The highest methane yield was obtained from biochemical methane potential (BMP) value based on lipid and lignin content at 5.27E $+10 \mathrm{NL} \mathrm{CH}_{4}$ digester $^{-1}$ day $^{-1}$. The results showed that livestock waste is a low-cost and extensive source of renewable energy in Iran that can be used optimally for biogas energy and electricity generation. Also the treatment of huge amount of livestock manure in anaerobic digestion is helpful for reducing its polluting effects on the environment.
\end{abstract}

Keywords: renewable energy, biogas, anaerobic digestion, organic waste, manure livestock

\section{Introduction}

Global demand for energy is increasing rapidly, because of population growth and technological advancements. Use of renewable energy sources is essential due to the limitation of fossil fuel sources and negative environmental effects (International Energy Agency, 2015; United Nations Environment Programme, 2014; Achinas and Euverink, 2016; Abdeshahian et al., 2016; Santos et al., 2018; Chandekar and Debnath, 2018). Biogas is one of the important renewable energy sources which is produced through decomposing organic waste under anaerobic conditions by microorganisms (Travnicek et al., 2018; Scarlat et al., 2018a; Angelidaki et al., 2018). Biogas is mainly composed of $\mathrm{CH}_{4}(60 \%)$ and $\mathrm{CO}_{2}$ (35-40\%) (Ilaboya et al., 2010; Sahota et al., 2018). Organic materials such as animal, human and plant wastes are biodegradable and can be converted into biogas (Zareei, 2018; Cu et al., 2015; Ozer, 2017; Yazan et al., 2018). Manure waste obtained from livestock industries are the largest and cheapest source for biogas production in anaerobic process (Comparetti et al., 2012; Ch'ng et al., 2014; Plume et al., 2012; Than, 2005; $\mathrm{Cu}$ et al., 2012; Yildirim et al., 2017; Mohammadi Maghanaki et al., 2013). Manure livestock is a type of organic waste which will be 


$$
-6500-
$$

hazardous to environment if it is not managed suitably. Livestock manure contains residues of some harmful substances such as growth hormones, antibiotics and heavy metals. So, disposal of them contaminates air, soil and water sources and prevalence of the human diseases (Abdeshahian et al., 2016; Pessuto et al., 2016). Anaerobic treatment of manure has the beneficial outcomes of reducing environmental pollution through proper waste management, reduction of unpleasant odors and microbial pathogens with a sustainable production of energy source as biogas (Wyman and Goodman, 1993; Mata-alvarez, 2000; Gebrezgabher et al., 2010; Holm-Nielsen et al., 2009; Wang et al., 2018; Samun et al., 2017; Neshat et al., 2017). Also it can be used for the production of a rich fertilizer which is favorable for the improvement of agricultural lands (De-Vries, 2012; Meyer et al., 2018; Eze and Agbo, 2010).

Considering the importance of animal waste as the enormous raw substances for energy production, many studies have been carried out on assessing the potential of biogas production from livestock waste. For instance, the potential of biogas production from livestock waste was studied in Turkey. The results showed that the amount of 2177.55 million $\mathrm{m}^{3}$ of biogas can be produced annually in Turkey (Onurbas-Avcioglu and Turker, 2012). Similar studies have also been performed in Finland, Sweden and Denmark to estimate the potential of energy production from livestock waste as evaluated in Turkey. These studies showed that the potential of biogas production is 332.97, 352.09 and 402.1 million $\mathrm{m}^{3} \mathrm{yr}^{-1}$ in mentioned countries, respectively (Abdeshahian et al., 2016; Luostarinen, 2013).

In recent years, the animal husbandry is drastically grown in Iran (Hamzeh et al., 2011; Government of Islamic Republic of Iran and Food and Agriculture Organization, 2012; Tehran Chamber of Commerce, Industries, Mines and Agriculture, 2016; Beldman et al., 2017). Number of industrial cattle farms in Iran based on capacity from 1990-2016 is shown in Figure 1.

No. of industrial cattle farms based on capacity in Iran from 1990-2016

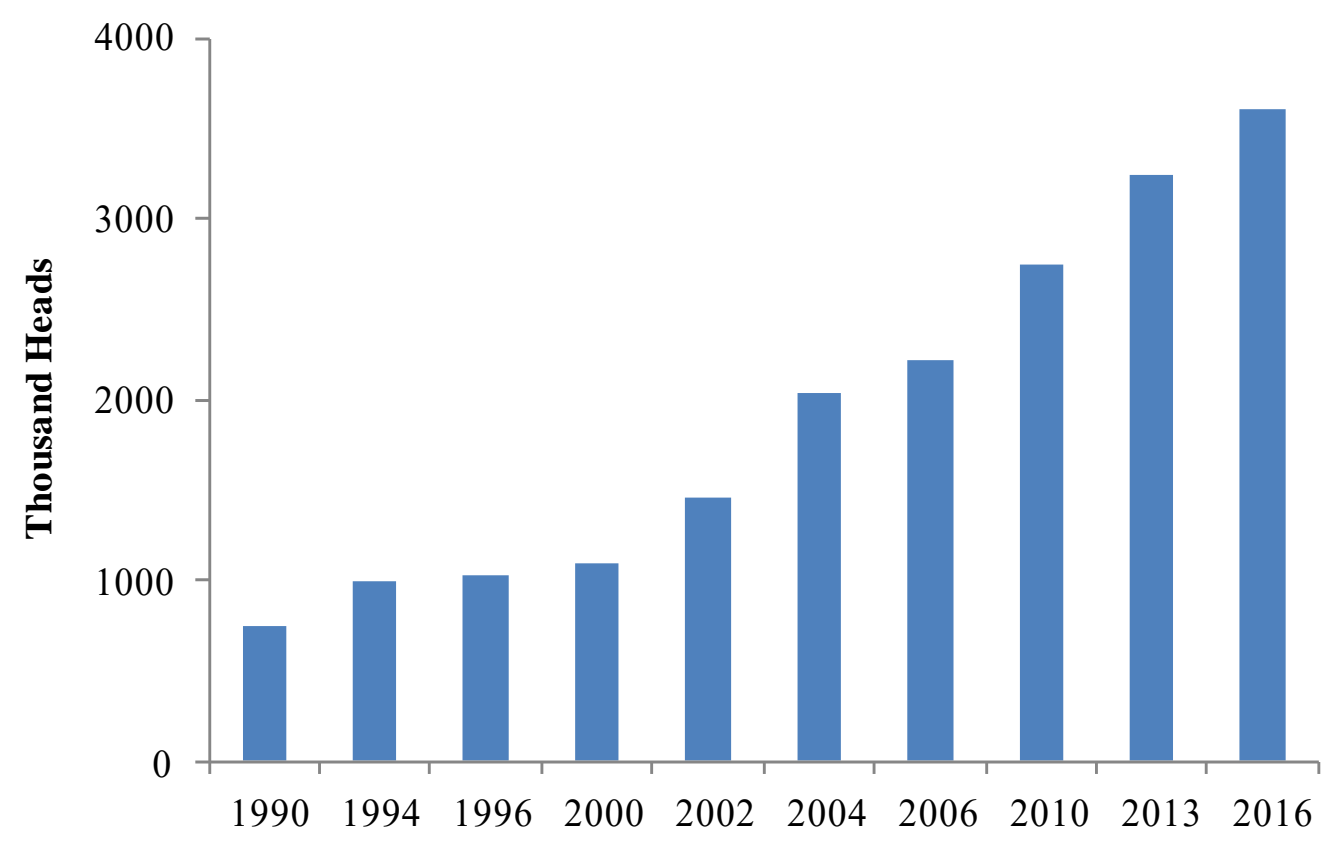

Figure 1. The increasing capacity of industrial cattle farms in Iran from 1990-2016 (Tehran Chamber of Commerce, Industries, Mines and Agriculture, 2016) 
As can be seen, industrial cattle farms capacity in Iran has revealed an increasing trend from 1990-2016. The increase population of the livestock has caused an elevated production of livestock waste, resulting in the difficulty with the disposal of a large amount of manure. Unsafe disposal of livestock waste and its accumulation in environment has caused unfavorable conditions due to creating a favorable environment for growth and spread of microbes, in addition to intolerable odor and insects (Zareei, 2018), but could instead be used as a tremendous source for generating biogas energy. A wide variety of calculations exist to evaluate the potential of biogas generation from livestock waste, each of them considers different parameters such as $\mathrm{pH}$, temperature, type, concentration and composition of raw materials and the time remaining in digester (Costa et al., 2016; Abdeshahian et al., 2016; Boysan et al., 2015; Cu et al., 2015; Burg et al., 2018; Zareei, 2018).

A wide variety of calculations exist to evaluate the potential of biogas generation from livestock waste. Some studies provide information about the factors that affect the biogas production such as $\mathrm{pH}$, temperature, type, concentration and composition of raw materials and the time remaining in digester (Costa et al., 2016; Abdeshahian et al., 2016; Boysan et al., 2015; $\mathrm{Cu}$ et al., 2015; Burg et al., 2018; Zareei, 2018). But variation of quantity of biogas generated from different evaluation methods has rarely been studied in a distinct study. The current study aims to compare the potential of biogas energy generation from the livestock waste produced from the animal husbandry in Iran using different calculation methods.

\section{Materials and methods}

\section{Livestock population in Iran}

In order to calculate the potential of biogas generation from the livestock manure, initial data was collected from the Statistical Centre of Iran (SCI) (Statistical Centre of Iran, 2017). According to the data reported by SCI, in 2016, Iran had a total of 26061 industrial cattle farms with a capacity of 3619696 heads. In this year 18547 cattle farms were active and the rest were inactive. The total number of cattle was 1439391 heads in Iran in 2016. Table 1 shows the number of specified groups of cattle population in each province of Iran.

Table 1. Number of specified groups of cattle population in Iran (Thousand Heads) (Statistical Centre of Iran, 2017)

\begin{tabular}{c|c|c|c|c|c|c}
\hline \multirow{2}{*}{ Province } & \multicolumn{2}{|c|}{ Original } & \multicolumn{2}{c|}{ Crossbred } & \multirow{2}{*}{ Native } & \multirow{2}{*}{ Total } \\
\cline { 2 - 6 } & Holstein & Other races & Holstein & Other races & & \\
\hline Azerbaijan, West & 21.98 & 0.52 & 3.13 & 2.26 & 0.45 & 28.36 \\
Azerbaijan, East & 10.83 & 0.88 & 0.78 & 0.16 & 0.55 & 13.21 \\
Ardabil & 21.48 & 0 & 2.99 & 0.41 & 0.06 & 24.95 \\
Isfahan & 125.94 & 1.24 & 72.95 & 6.6 & 0.12 & 206.87 \\
Alborz & 66.77 & 0.27 & 3.30 & 1.19 & 0.76 & 72.31 \\
Ilam & 5.61 & 0.10 & 0.43 & 0 & 0 & 6.15 \\
Bushehr & 0.44 & 0.03 & 1.88 & 5.14 & 2.02 & 9.52 \\
Tehran & 223.55 & 5.84 & 14.81 & 38.31 & 38.88 & 321.42 \\
ChaharMahaal and Bakhtiari & 28.98 & 0.10 & 0.32 & 0.01 & 0 & 29.42 \\
Khorasan, South & 8.93 & 0.03 & 2.10 & 0.41 & 0 & 11.49 \\
\hline
\end{tabular}




$$
-6502-
$$

Table 1 (continued). Number of specified groups of cattle population in Iran (Thousand Heads) (Statistical Centre of Iran, 2017)

\begin{tabular}{c|c|c|c|c|c|c}
\hline \multirow{2}{*}{ Province } & \multicolumn{2}{|c|}{ Original } & \multicolumn{2}{c|}{ Crossbred } & \multirow{2}{*}{ Native } & \multirow{2}{*}{ Total } \\
\cline { 2 - 6 } & Holstein & Other races & Holstein & Other races & & \\
\hline Khorasan, Razavi & 92.64 & 2.75 & 16.01 & 1.17 & 0.03 & 112.62 \\
Khorasan, North & 4.75 & 0.23 & 2.46 & 0.03 & 0.14 & 7.62 \\
Khuzestan & 4.75 & 0.37 & 15.35 & 2.28 & 0.43 & 23.19 \\
Zanjan & 14.19 & 0 & 2.63 & 0.04 & 0.14 & 17.02 \\
Semnan & 16.84 & 4.06 & 12.56 & 23.38 & 0.78 & 57.64 \\
Sistan and Baluchestan & 2.19 & 0.23 & 0.29 & 0.58 & 0.79 & 4.11 \\
Fars & 75.65 & 0.37 & 17.86 & 8.21 & 3.00 & 105.12 \\
Qazvin & 65.59 & 0.10 & 5.84 & 1.05 & 0 & 72.59 \\
Qom & 28.73 & 1.13 & 5.56 & 16.75 & 12.65 & 64.84 \\
Kurdistan & 1.85 & 0.47 & 0.38 & 0.31 & 0.40 & 3.42 \\
Kerman & 29.28 & 0.26 & 2.35 & 0.53 & 0.75 & 33.19 \\
Kermanshah & 14.74 & 1.14 & 0.33 & 0.06 & 0.25 & 16.54 \\
Kohgiluyeh and Boyer-Ahmad & 3.13 & 0 & 1.28 & 0.02 & 0 & 4.43 \\
Golestan & 15.35 & 1.56 & 0.98 & 0.35 & 0.01 & 18.26 \\
Gilan & 2.31 & 0.22 & 0.41 & 0.10 & 0.70 & 3.76 \\
Lorestan & 6.35 & 0 & 2.21 & 0.30 & 1.01 & 9.89 \\
Mazandaran & 10.87 & 0.82 & 2.90 & 6.39 & 0.66 & 21.65 \\
Markazi & 10.62 & 0 & 15.13 & 33.95 & 0.34 & 60.05 \\
Hormozgan & 0.24 & 0.01 & 0.14 & 0.52 & 0.34 & 1.27 \\
Hamadan & 15.34 & 0.25 & 1.62 & 0.29 & 0 & 17.52 \\
Yazd & 18.35 & 0.37 & 38.89 & 2.96 & 0.24 & 60.83 \\
Iran & 948.42 & 23.48 & 248.02 & 153.85 & 65.6 & 1439.39 \\
\hline
\end{tabular}

\section{Livestock manure production}

Livestock waste is composed of the organic matter that can be treated as the potential raw substance for the production of bioenergy (Afazeli et al., 2014; Mathias, 2014). The amount of livestock manure can vary based on the type of animal, feeding methods, animal body size, the type of breeding and keeping time at day or night (OnurbasAvcioglu and Turker, 2012; Omrani, 1996). Abdeshahian et al., (2016) categorized cattle into the large ruminants and calculated the amount of livestock manure based on the live body weight. The amount of the manure was calculated based on the $9 \%$ of body weight for large ruminants. The average live body weight accounted $250 \mathrm{~kg}$ for the large ruminants. Accordingly, the average amount of the manure was calculated based on $22.5 \mathrm{~kg} /$ day for the large ruminants. This is while that Boysan et al. (2015) calculated the amount of livestock manure production based on $10 \mathrm{~kg} /$ day for one cattle. On the other hand, Plume et al. (2012) calculated the manure output from livestock in a year using Equation 1.

$$
M=\sum_{n=1}^{i} N_{i} * M_{i}
$$




$$
-6503-
$$

where:

M- Livestock manure produced in region ( $\mathrm{t}$ ),

$\mathrm{n}$ - Number of specified groups of livestock population in region,

$\mathrm{N}_{\mathrm{i}}$ - Average number of livestock present year-round within $\mathrm{i}^{\text {th }}$ group of livestock,

$\mathrm{m}_{\mathrm{i}}$ - Manure produced per one head in a year in the $\mathrm{i}^{\mathrm{th}}$ group of livestock $(\mathrm{t})$.

Furthermore Zareei (2018) denoted that the livestock manure production can be calculated using parameters including livestock weight and the ratio of the annual manure generation to livestock weight as shown in Table 2 .

Table 2. Coefficient for calculation of manure production from livestock (Zareei, 2018)

\begin{tabular}{c|c|c}
\hline Material type & Livestock weight & The ratio of the annual livestock manure to livestock weight \\
\hline Cow manure & $500-620$ & 2.6 \\
\hline
\end{tabular}

The average weight of livestock was estimated according to the dominant races of the area. As stated in Table 2, livestock weight was considered in the range of 500 to 620 . The total amount of livestock waste can be calculated by multiplying the waste mass by the population number (Zareei, 2018).

\section{The potential of biogas production from the livestock manure}

The biogas produced from the livestock waste is affected by the different factors such as feeding regime, animal type, body weight, the proportion of total solids and the waste availability (United Nations Environment Programme, 2014; Than, 2005). Abdeshahian et al. (2016) considered the total solids of the waste as an important factor for the production of biogas from the livestock waste. They considered the total solids value of livestock manure as $25 \%$ for the large ruminants with the quantity of estimated biogas produced per kilogram of the total solids as $0.6(0.6 \mathrm{~m} 3 / \mathrm{kg}$ TS $)$. Also they have pointed that the collection of the manure cannot always be carried out efficiently for the production of biogas and the availability of the manure is varied. Hence, for the calculating of biogas production from the livestock manure the availability coefficient was considered as 50\% for large ruminants (Onurbas-Avcioglu and Turker, 2012; Afazeli et al., 2014). By taking into account the mentioned assumptions, the theoretical potential of biogas production from the livestock manure and the potential of electricity generation from the biogas was calculated as shown in Equations 2 and 3 (Abdeshahian et al., 2016).

$$
T P B=M * T S * A C * E B_{T S}
$$

where:

TPB- Theoretical potential of biogas $\left(\mathrm{m}^{3} \mathrm{yr}^{-1}\right)$,

M- Total amount of the manure produced for each region $\left(\mathrm{kg} \mathrm{yr}^{-1}\right)$,

TS- Ratio of the total solids of the animal manure,

AC- Availability coefficient,

EBTS- Quantity of estimated biogas produced per kilogram of the total solids $\left(\mathrm{m}^{3} \mathrm{~kg}^{-1}\right.$ TS).

$$
e_{\text {biogas }}=E_{\text {biogas }} * n
$$


where:

$\mathrm{e}_{\text {biogas- }}$ Quantity of generated electricity $\left(\mathrm{kWh} \mathrm{yr}^{-1}\right)$,

$\mathrm{E}_{\text {biogas }}$ - Unconverted raw energy in the biogas $\left(\mathrm{kWh} \mathrm{yr}^{-1}\right)$,

$n$ - Overall efficiency of the conversion of biogas to electricity (\%). The amount of $n$ is varied depending on the power generation plants. The $n$ value is considered $35-42 \%$ and $25 \%$ in the power plants with large turbine system and small generators, respectively (Hosseini and Wahid, 2014; Benito et al., 2015). In this study, the $n$ value was assumed as 30\% based on Iran power plants characterization (Iran Energy Ministry, 2017).

The quantity of $\mathrm{E}_{\text {biogas }}$ is calculated using Equation 4 (Abdeshahian, 2016).

$$
E_{\text {biogas }}=\text { Energycontent }_{\text {biogas }} * m_{\text {biogas }}
$$

where:

Energy content biogas $^{-}$Calorific value of biogas $\left(\mathrm{kWh} \mathrm{m}^{-3}\right)$. The quantity of the Energy content $_{\text {biogas }}$ is assumed as $6 \mathrm{kWh} \mathrm{m}^{-3}$ by considering the biogas calorific value as $21.5 \mathrm{MJ}$ per $\mathrm{m}^{3}$ biogas $(1 \mathrm{kWh}=3.6 \mathrm{MJ})$ (Hosseini and Wahid, 2014; Garcia, 2014).

$\mathrm{m}_{\text {biogas }}$ Amount of biogas produced per year $\left(\mathrm{m}^{3} \mathrm{yr}^{-1}\right)$.

On the other hand, Boysan et al. (2015) calculated the potential of biogas production from livestock manure based on $0.33 \mathrm{~m}^{3} /$ cattle with considering assumptions including fermenter temperature as $37^{\circ} \mathrm{C}, 20 \%$ mass in manure and waiting time as 30 days. It is while that in the study conducted by Zareei (2018) the biogas production potential per kilogram of cow manure was considered as $0.28-0.28 \mathrm{~m}^{3}$. Furthermore, Plume et al. (2012) proposed Equation 5 for calculating the potential of biogas production from livestock manure.

$$
V_{B}=\sum_{n}^{i} N_{i} * m_{i} * K_{D M i} * K_{O M i} * v_{B i}
$$

where:

$\mathrm{V}_{\mathrm{B}}$ - Biogas volume, potentially obtainable from manure biomass in region in a year $\left(\mathrm{m}^{3}\right)$, $\mathrm{K}_{\mathrm{DMi}}$ - Dry matter content in manure produced by $i^{\text {th }}$ group of animals in region,

$\mathrm{K}_{\text {Omi- }}$ Organic matter content in dry matter of manure produced by $\mathrm{i}^{\text {th }}$ group of animals in region,

$\mathrm{v}_{\mathrm{Bi}}$ - Specific biogas output from manure organic matter for $i^{\text {th }}$ group of animals in region $\left(\mathrm{m}^{3} \mathrm{t}^{-1}\right)$.

The energy of biogas obtainable from manure biomass in region then was calculated according to Equation 6 (Plume et al., 2012).

$$
E_{B}=\sum_{n=1}^{i} N_{i} * m_{i} * K_{D M i} * K_{O M i} * v_{B i} * e_{B i}
$$

where:

$\mathrm{E}_{\mathrm{B}}$ - Energy potential obtainable from biogas produced from manure $(\mathrm{kWh})$,

$\mathrm{e}_{\mathrm{Bi}}$ - Specific heat energy content of biogas obtained from manure produced by $i^{\text {th }}$ group of animals $\left(\mathrm{kWh} \mathrm{m}^{-3}\right)$. 


$$
-6505-
$$

\section{Calculation of methane $\left(\mathrm{CH}_{4}\right)$ content of biogas}

The biogas obtained from the anaerobic digestion process of livestock manure is composed of 50-70\% of methane (Omar et al., 2009; Nasir et al., 2012; Ounnar et al., 2012; Nasir et al., 2013). The methane yield can be calculated based on the original Hashimoto equation (Eq. 7 below) (Chen and Hashimoto, 1978; Hashimoto et al., 1981).

$$
\gamma=\frac{\beta_{0} * S_{0}}{H R T}\left(1-\frac{K}{H R T * \mu m-1+K}\right)
$$

where:

$\gamma$ - Methane yield ( $\mathrm{NL} \mathrm{CH}_{4}$ digester $^{-1}$ day $\left.^{-1}\right)$,

$\beta_{\mathrm{a}}$ - Biochemical methane production (BMP) value of specific substrate $\left(\mathrm{NL} \mathrm{CH}_{4} \mathrm{~kg} \mathrm{VS}^{-1}\right)$, $S_{\mathrm{a}}$ - VS concentration $\left(\mathrm{g} \mathrm{kg}^{-1}\right)$,

HRT- Hydraulic retention time (days),

$\mathrm{K}$ - Kinetic constant $\left(\mathrm{K}=0.6+0.0206\right.$.EXP $\left[0.051 . \mathrm{S}_{0}\right]$,

$\mu m$ - Maximum specific growth rate $\left(\right.$ day $\left.^{-1}\right), \mu \mathrm{m}=0.013 \mathrm{~T}-0.129$, for temperature $(\mathrm{T})$ between 20 and $60{ }^{\circ} \mathrm{C}$.

Rennuit and Sommer (2013) extended the Hashimoto equation to calculate the methane yield for a biogas digester. The extended equation takes into account the difference in lowland and highland temperature regimes. It is assumed that the mean monthly air temperature represents the mean monthly digester temperature (Perrigault et al., 2012). Hence, the methane yield for a biogas digester for an air temperature between 15 to $30{ }^{\circ} \mathrm{C}$ and 20 to $60{ }^{\circ} \mathrm{C}$ is calculated based on Equations 8 and 9, respectively (Rennuit and Sommer, 2013).

$$
\begin{gathered}
\gamma_{\text {winter }}(H R T, \mu m, T D)=\beta_{0} *\left[1-\frac{K}{\mu m(T D) *\left(H R T+\frac{1}{\mu m(T D)}\right)-1+K}\right] \\
\gamma_{\text {summer }}(H R T, \mu m, T D)=\beta_{0} *\left[1-\frac{K}{\mu m(T D) * H R T-1+K}\right]
\end{gathered}
$$

where:

$\mu \mathrm{m}(\mathrm{Td})$ - maximum specific growth rate of the microorganisms at the digester temperature in the temperature interval from 10 to $30{ }^{\circ} \mathrm{C}\left(\mu \mathrm{m}[\mathrm{Td}]=0.0039 \mathrm{e}^{0.1188 \cdot(\mathrm{Td})}\right.$ $\left(\right.$ day $\left.^{-1}\right)$ and (Td) is the digester temperature which is set to be equal to the average monthly air temperature $\left({ }^{\circ} \mathrm{C}\right)$ represented by region.

In line with this, $\mathrm{Cu}$ et al. (2015) suggested that the BMP value ( $\beta_{0}$ in the Hashimoto equation) from different types of substrate can be predicted based on their chemical composition. Biogas production is related to the chemical composition of the substrate. Low concentrations of organic matter such as lipids and protein will lead to a low biogas production. However, the high concentration of organic matter in substrates will have a negative effect on biogas production, causing foaming and inhibition if not cofermented with biomasses low in protein and lipids ( $\mathrm{Cu}$ et al., 2015; Kougias et al., 
2013). There is a clear relationship between lipid content of the biomass and biochemical methane potential. Lipid content is the most important factor for biochemical methane potential compared to other components. However, in a biogas technology every component in the substrates affects biogas production. It was found that lipid, lignin, protein, and cellulose contents were the main chemical components of substrates contributing to the variation in the BMP $(\mathrm{Cu}$ et al., 2015). Equations to predict BMP are shown in Table 3.

Table 3. Equations to predict BMP for livestock manure (Cu et al., 2015)

\begin{tabular}{c|c}
\hline Variables & Equations for BMP \\
\hline Lipid & $57.9+35 \times$ lipid \\
Lipid, lignin & $186+30.6 \times$ lipid $-5.13 \times$ lignin \\
Lipid, lignin, dry matter & $167+30.1 \times$ lipid $-5.43 \times$ lignin $+1.15 \times$ dry matter \\
Lipid, lignin, cellulose & $201+31.5 \times$ lipid $-3.85 \times$ lignin $-1.88 \times$ cellulose \\
\hline
\end{tabular}

\section{Results and discussion}

\section{Calculation of livestock manure production in Iran}

The amount of livestock manure production was calculated for all provinces of Iran based on Abdeshahian, Boysan and Zareei methods. The results are shown in Table 4.

Table 4. The estimated values of livestock manure of different provinces in Iran in 2016 based on different methods

\begin{tabular}{|c|c|c|c|c|c|c|c|}
\hline \multicolumn{8}{|c|}{ Manure production $\left({\left.\mathrm{t} \mathrm{yr}^{-1}\right)}^{-1}\right.$} \\
\hline Province & Abdeshahian & Boysan & Zareei & Province & Abdeshahian & Boysan & Zareei \\
\hline $\begin{array}{c}\text { Azerbaijan, } \\
\text { West }\end{array}$ & $2.33 \mathrm{E}+05$ & $1.04 \mathrm{E}+05$ & $4.13 \mathrm{E}+04$ & Fars & $8.63 \mathrm{E}+05$ & $3.84 \mathrm{E}+05$ & $1.53 \mathrm{E}+05$ \\
\hline $\begin{array}{c}\text { Azerbaijan, } \\
\text { East }\end{array}$ & $1.08 \mathrm{E}+05$ & $4.82 \mathrm{E}+04$ & $1.92 \mathrm{E}+04$ & Qazvin & $5.96 \mathrm{E}+05$ & $2.65 \mathrm{E}+05$ & $1.06 \mathrm{E}+05$ \\
\hline Ardabil & $2.05 \mathrm{E}+05$ & $9.11 \mathrm{E}+04$ & $3.63 \mathrm{E}+04$ & Qom & $5.33 \mathrm{E}+05$ & $2.37 \mathrm{E}+05$ & $9.44 \mathrm{E}+04$ \\
\hline Isfahan & $1.70 \mathrm{E}+06$ & $7.55 \mathrm{E}+05$ & $3.01 \mathrm{E}+05$ & Kurdistan & $2.82 \mathrm{E}+04$ & $1.25 \mathrm{E}+04$ & $4.99 \mathrm{E}+03$ \\
\hline Alborz & $5.94 \mathrm{E}+05$ & $2.64 \mathrm{E}+05$ & $1.05 \mathrm{E}+05$ & Kerman & $2.73 \mathrm{E}+05$ & $1.21 \mathrm{E}+05$ & $4.83 \mathrm{E}+04$ \\
\hline Ilam & $5.05 \mathrm{E}+04$ & $2.25 \mathrm{E}+04$ & $8.96 \mathrm{E}+03$ & Kermanshah & $1.36 \mathrm{E}+05$ & $6.04 \mathrm{E}+04$ & $2.41 \mathrm{E}+04$ \\
\hline Bushehr & $7.82 \mathrm{E}+04$ & $3.48 \mathrm{E}+04$ & $1.39 \mathrm{E}+04$ & $\begin{array}{c}\text { Kohgiluyeh } \\
\text { and Boyer- } \\
\text { Ahmad }\end{array}$ & $3.64 \mathrm{E}+04$ & $1.62 \mathrm{E}+04$ & $6.46 \mathrm{E}+03$ \\
\hline Tehran & $2.64 \mathrm{E}+06$ & $1.17 \mathrm{E}+06$ & $4.68 \mathrm{E}+05$ & Golestan & $1.50 \mathrm{E}+05$ & $6.67 \mathrm{E}+04$ & $2.66 \mathrm{E}+04$ \\
\hline $\begin{array}{l}\text { ChaharMahaal } \\
\text { and Bakhtiari }\end{array}$ & $2.42 \mathrm{E}+05$ & $1.07 \mathrm{E}+05$ & $4.28 \mathrm{E}+04$ & Gilan & $3.10 \mathrm{E}+04$ & $1.38 \mathrm{E}+04$ & $5.49 \mathrm{E}+03$ \\
\hline $\begin{array}{l}\text { Khorasan, } \\
\text { South }\end{array}$ & $9.44 \mathrm{E}+04$ & 4.19E+04 & $1.67 \mathrm{E}+04$ & Lorestan & $8.12 \mathrm{E}+04$ & $3.61 \mathrm{E}+04$ & $1.44 \mathrm{E}+04$ \\
\hline $\begin{array}{l}\text { Khorasan, } \\
\text { Razavi }\end{array}$ & $9.25 \mathrm{E}+05$ & $4.11 \mathrm{E}+05$ & $1.64 \mathrm{E}+05$ & Mazandaran & $1.78 \mathrm{E}+05$ & $7.90 \mathrm{E}+04$ & $3.15 \mathrm{E}+04$ \\
\hline $\begin{array}{l}\text { Khorasan, } \\
\text { North }\end{array}$ & $6.26 \mathrm{E}+04$ & $2.78 \mathrm{E}+04$ & $1.11 \mathrm{E}+04$ & Markazi & $4.93 \mathrm{E}+05$ & $2.19 \mathrm{E}+05$ & $8.74 \mathrm{E}+04$ \\
\hline Khuzestan & $1.90 \mathrm{E}+05$ & $8.46 \mathrm{E}+04$ & $3.38 \mathrm{E}+04$ & Hormozgan & $1.04 \mathrm{E}+04$ & $4.64 \mathrm{E}+03$ & $1.85 \mathrm{E}+03$ \\
\hline Zanjan & $1.40 \mathrm{E}+05$ & $6.21 \mathrm{E}+04$ & $2.48 \mathrm{E}+04$ & Hamadan & $1.44 \mathrm{E}+05$ & $6.40 \mathrm{E}+04$ & $2.55 \mathrm{E}+04$ \\
\hline
\end{tabular}




$$
-6507 \text { - }
$$

Table 4 (continued). The estimated values of livestock manure of different provinces in Iran in 2016 based on different methods

\begin{tabular}{c|c|c|c|c|c|c|c}
\hline \multicolumn{1}{c}{ Manure production $\left(\mathbf{t ~ y r}^{-\mathbf{1}}\right)$} \\
\hline Province & Abdeshahian & Boysan & Zareei & Province & Abdeshahian & Boysan & Zareei \\
\hline Semnan & $4.73 \mathrm{E}+05$ & $2.10 \mathrm{E}+05$ & $8.39 \mathrm{E}+04$ & Yazd & $5.00 \mathrm{E}+05$ & $2.22 \mathrm{E}+05$ & $8.86 \mathrm{E}+04$ \\
Sistan and & $3.38 \mathrm{E}+04$ & $1.50 \mathrm{E}+04$ & $5.99 \mathrm{E}+03$ & Iran & $1.18 \mathrm{E}+07$ & $5.25 \mathrm{E}+06$ & $2.10 \mathrm{E}+06$ \\
Baluchestan & & & &
\end{tabular}

Manure production by livestock in different groups based on Plume et al. (2012) is presented in Table 5.

Table 5. Manure production by livestock in different groups based on Plume et al. (2012)

\begin{tabular}{c|c}
\hline Group characteristics & Manure production per head $\left.\mathbf{t} \mathbf{~ y r}^{-\mathbf{1}}\right)$ \\
\hline Dairy cows & $0.0024 \mathrm{Y}_{\mathrm{D}}+0.447$ \\
Heifers & 8 \\
Calves & 2.6 \\
Cattle & 12 \\
\hline
\end{tabular}

Average values of manure production per one head in a year for all groups of livestock, except dairy cows, are given in Table 5. Average manure production per one dairy cow is dependent on cow's milk yield and calculates help by regression Equation 10 (Plume et al., 2012).

$$
m_{1}=0.0024 * Y_{d}+0.447
$$

where:

$\mathrm{m}_{1}$ - Average manure production per dairy cow in region in a year $\left(\mathrm{t} \mathrm{yr}^{-1}\right)$, $\mathrm{Y}_{\mathrm{d}}$ - Average milk yield per dairy cow in region in a year $\left(\mathrm{kg} \mathrm{yr}^{-1}\right)$.

Aiming to improve accuracy of manure resources evaluation, all the animals was divided in 4 groups (dairy cows, heifers, calves and cattle) according to its manure production capability per one head. The number of livestock within specified groups in each province of Iran in 2016 is presented in Table 6.

Table 6. Number of livestock within specified groups in each province of Iran in 2016

\begin{tabular}{c|c|c|c|c|c|c|c|c|c}
\hline \multirow{2}{*}{ Province } & \multicolumn{3}{|c|}{ Dairy cow } & \multicolumn{2}{c|}{ Heifers } & \multicolumn{2}{c|}{ Calves } & \multicolumn{2}{c}{ Cattle } \\
\cline { 2 - 10 } & $\begin{array}{c}\text { Lactating } \\
\text { cow }\end{array}$ & \multicolumn{2}{|c|}{ Dry cow } & Pregnant & Unpregnant & Bull & Cow & $\begin{array}{c}\text { Breeding } \\
\text { cattle }\end{array}$ & Other \\
\hline $\begin{array}{c}\text { Azerbaijan, } \\
\text { West }\end{array}$ & 9085 & 2197 & 748 & 3175 & 2461 & 4016 & 2881 & 1478 & 2322 \\
$\begin{array}{c}\text { Azerbaijan, } \\
\text { East }\end{array}$ & 3804 & 1069 & 560 & 1154 & 971 & 2629 & 1600 & 940 & 482 \\
Ardabil & 7977 & 1964 & 396 & 2184 & 1841 & 4231 & 4286 & 389 & 1688 \\
Isfahan & 85333 & 18747 & 6047 & 25458 & 16938 & 22081 & 25890 & 1615 & 4766 \\
Alborz & 28921 & 6951 & 2041 & 7956 & 6450 & 5529 & 11199 & 915 & 2350 \\
Ilam & 1552 & 853 & 704 & 315 & 322 & 1340 & 344 & 438 & 285 \\
\hline
\end{tabular}


Table 6 (continued). Number of livestock within specified groups in each province of Iran in 2016

\begin{tabular}{|c|c|c|c|c|c|c|c|c|c|}
\hline \multirow{3}{*}{ Province } & \multicolumn{3}{|c|}{ Dairy cow } & \multicolumn{2}{|c|}{ Heifers } & \multicolumn{2}{|c|}{ Calves } & \multicolumn{2}{|c|}{ Cattle } \\
\hline & \multirow{2}{*}{$\begin{array}{c}\text { Lactating } \\
\text { cow }\end{array}$} & \multicolumn{2}{|c|}{ Dry cow } & \multirow{2}{*}{ Pregnant } & \multirow{2}{*}{ Unpregnant } & \multirow{2}{*}{ Bull } & \multirow{2}{*}{ Cow } & \multirow{2}{*}{$\begin{array}{c}\text { Breeding } \\
\text { cattle }\end{array}$} & \multirow{2}{*}{ Other } \\
\hline & & Pregnant & Unpregnant & & & & & & \\
\hline Bushehr & 1026 & 609 & 381 & 346 & 308 & 6335 & 151 & 53 & 316 \\
\hline Tehran & 93015 & 25956 & 10066 & 25284 & 24227 & 104542 & 28958 & 6220 & 3152 \\
\hline $\begin{array}{l}\text { ChaharMahaal } \\
\text { and Bakhtiari }\end{array}$ & 10136 & 3738 & 2031 & 3230 & 2535 & 2896 & 3848 & 972 & 41 \\
\hline $\begin{array}{l}\text { Khorasan, } \\
\text { South }\end{array}$ & 3451 & 1109 & 746 & 1027 & 1226 & 1573 & 1552 & 437 & 371 \\
\hline $\begin{array}{c}\text { Khorasan, } \\
\text { Razavi }\end{array}$ & 42617 & 10634 & 3539 & 10815 & 11499 & 13083 & 14029 & 931 & 5475 \\
\hline $\begin{array}{l}\text { Khorasan, } \\
\text { North }\end{array}$ & 2290 & 618 & 173 & 691 & 546 & 1707 & 945 & 592 & 66 \\
\hline Khuzestan & 3881 & 1480 & 362 & 576 & 1021 & 11323 & 1106 & 676 & 2767 \\
\hline Zanjan & 5984 & 1752 & 282 & 2081 & 2004 & 1391 & 2074 & 1284 & 174 \\
\hline Semnan & 17947 & 3811 & 1592 & 4081 & 3311 & 10407 & 3722 & 6540 & 6233 \\
\hline $\begin{array}{l}\text { Sistan and } \\
\text { Baluchestan }\end{array}$ & 627 & 404 & 136 & 177 & 107 & 1322 & 256 & 290 & 794 \\
\hline Fars & 33313 & 9111 & 2447 & 10681 & 11417 & 18293 & 11255 & 3097 & 5514 \\
\hline Qazvin & 26322 & 6201 & 658 & 7380 & 5975 & 11451 & 10288 & 596 & 3722 \\
\hline Qom & 9559 & 2301 & 745 & 2928 & 2470 & 36488 & 3770 & 745 & 5835 \\
\hline Kurdistan & 1090 & 206 & 32 & 278 & 242 & 1069 & 281 & 130 & 100 \\
\hline Kerman & 10349 & 3422 & 1581 & 3723 & 2867 & 4525 & 3462 & 2925 & 338 \\
\hline Kermanshah & 5915 & 1134 & 978 & 1785 & 1701 & 1445 & 1973 & 644 & 971 \\
\hline $\begin{array}{c}\text { Kohgiluyeh } \\
\text { and Boyer- } \\
\text { Ahmad }\end{array}$ & 1301 & 358 & 17 & 438 & 471 & 1243 & 576 & 32 & 0 \\
\hline Golestan & 5635 & 2287 & 733 & 2333 & 2213 & 2361 & 2213 & 393 & 96 \\
\hline Gilan & 1204 & 310 & 235 & 575 & 241 & 390 & 547 & 250 & 19 \\
\hline Lorestan & 2848 & 818 & 116 & 958 & 598 & 2800 & 1573 & 29 & 153 \\
\hline Mazandaran & 7544 & 2354 & 689 & 2174 & 1922 & 2667 & 2348 & 195 & 1759 \\
\hline Markazi & 9822 & 2133 & 997 & 2387 & 1894 & 33381 & 5686 & 1367 & 2390 \\
\hline Hormozgan & 227 & 76 & 32 & 43 & 15 & 298 & 90 & 397 & 92 \\
\hline Hamadan & 5878 & 1999 & 922 & 1351 & 1197 & 3323 & 2248 & 606 & 0 \\
\hline Yazd & 21136 & 7208 & 1154 & 7487 & 5981 & 6754 & 7605 & 1526 & 1985 \\
\hline Iran & 459789 & 121810 & 41140 & 133071 & 114971 & 320893 & 156756 & 36702 & 54256 \\
\hline
\end{tabular}

In line with this, the amount of livestock manure production in different provinces of Iran was calculated based on Plume et al. (2012) and is presented in Table 7. 


$$
-6509-
$$

Table 7. The estimated values of livestock manure of different provinces in Iran in 2016 based on Plume et al. (2012)

\begin{tabular}{|c|c|c|c|c|c|c|}
\hline \multicolumn{7}{|c|}{ Manure production $\left({\left.\mathrm{t} \mathrm{yr}^{-1}\right)}^{-1}\right.$} \\
\hline \multirow{2}{*}{ Province } & \multicolumn{2}{|c|}{ Dairy cows } & \multirow{2}{*}{ Heifers } & \multirow{2}{*}{ Calves } & \multirow{2}{*}{ Cattle } & \multirow{2}{*}{ Total } \\
\hline & Lactating cow & Dry cow & & & & \\
\hline Azerbaijan, West & 199870 & 35340 & 45088 & 17932 & 45600 & 343830 \\
\hline Azerbaijan, East & 72276 & 19548 & 17000 & 10995 & 17064 & 136883 \\
\hline Ardabil & 127632 & 28320 & 32200 & 22144 & 24924 & 235220 \\
\hline Isfahan & 2218658 & 297528 & 339168 & 124724 & 76572 & 3056650 \\
\hline Alborz & 607341 & 107904 & 115248 & 43492 & 39180 & 913165 \\
\hline Ilam & 27936 & 18684 & 5096 & 4378 & 8676 & 64770 \\
\hline Bushehr & 4104 & 11880 & 5232 & 16863 & 4428 & 42507 \\
\hline Tehran & 1581255 & 432264 & 396088 & 347100 & 112464 & 2869171 \\
\hline ChaharMahaal and Bakhtiari & 233128 & 69228 & 46120 & 17534 & 12156 & 378166 \\
\hline Khorasan, South & 72471 & 22260 & 18024 & 8125 & 9696 & 130576 \\
\hline Khorasan, Razavi & 980191 & 170076 & 178512 & 70491 & 76872 & 1476142 \\
\hline Khorasan, North & 48090 & 9492 & 9896 & 6895 & 7896 & 82269 \\
\hline Khuzestan & 62096 & 22104 & 12776 & 32315 & 41316 & 170607 \\
\hline Zanjan & 155584 & 24408 & 32680 & 9009 & 17496 & 239177 \\
\hline Semnan & 287152 & 64836 & 59136 & 36735 & 153276 & 601135 \\
\hline Sistan and Baluchestan & 8151 & 6480 & 2272 & 4102 & 13008 & 34013 \\
\hline Fars & 732886 & 138696 & 176784 & 76824 & 103332 & 1228522 \\
\hline Qazvin & 605406 & 82308 & 106840 & 56521 & 51816 & 902891 \\
\hline Qom & 248534 & 36552 & 43184 & 104670 & 78960 & 511900 \\
\hline Kurdistan & 22890 & 2856 & 4160 & 3510 & 2760 & 36176 \\
\hline Kerman & 196631 & 60036 & 52720 & 20766 & 39156 & 369309 \\
\hline Kermanshah & 159705 & 25344 & 27888 & 8886 & 19380 & 241203 \\
\hline Kohgiluyeh and Boyer-Ahmad & 33826 & 4500 & 7272 & 4729 & 384 & 50711 \\
\hline Golestan & 118335 & 36240 & 36368 & 11892 & 5868 & 208703 \\
\hline Gilan & 20468 & 6540 & 6528 & 2436 & 3228 & 39200 \\
\hline Lorestan & 48416 & 11208 & 12448 & 11369 & 2184 & 85625 \\
\hline Mazandaran & 181056 & 36516 & 32768 & 13039 & 23448 & 286827 \\
\hline Markazi & 157152 & 37560 & 34248 & 101574 & 45084 & 375618 \\
\hline Hormozgan & 2043 & 1296 & 464 & 1008 & 5868 & 10679 \\
\hline Hamadan & 141072 & 35052 & 20384 & 14484 & 7272 & 218264 \\
\hline Yazd & 422720 & 100344 & 107744 & 37333 & 42132 & 710273 \\
\hline Iran & 9777075 & 1955400 & 1984336 & 1241875 & 1091496 & 16050182 \\
\hline
\end{tabular}

\section{Calculation of the potential of biogas production from the livestock manure in Iran}

The biogas production potential from the livestock manure in all provinces of Iran was calculated based on Abdeshahian, Boysan and Zareei methods and is presented in Table 8. 


$$
-6510-
$$

Table 8. The estimated values of biogas production in all provinces in Iran in 2016 based on different methods

\begin{tabular}{|c|c|c|c|c|c|c|c|}
\hline \multicolumn{8}{|c|}{ Biogas production potential (million $\mathrm{m}^{3} \mathrm{yr}^{-1}$ ) } \\
\hline Province & Abdeshahian & Boysan & Zareei & Province & Abdeshahian & Boysan & Zareei \\
\hline $\begin{array}{l}\text { Azerbaijan, } \\
\text { West }\end{array}$ & 17.46 & 3.41 & 11.15 & Fars & 64.75 & 12.66 & 41.32 \\
\hline $\begin{array}{l}\text { Azerbaijan, } \\
\text { East }\end{array}$ & 8.13 & 1.59 & 5.19 & Qazvin & 44.71 & 8.74 & 28.53 \\
\hline Ardabil & 15.37 & 3.00 & 9.81 & Qom & 39.93 & 7.80 & 25.49 \\
\hline Isfahan & 127.42 & 24.91 & 81.32 & Kurdistan & 2.11 & 0.41 & 1.34 \\
\hline Alborz & 44.53 & 8.70 & 28.4269 & Kerman & 20.44 & 3.99 & 13.04 \\
\hline Ilam & 3.78 & 0.74 & 2.41 & Kermanshah & 10.19 & 1.99 & 6.50 \\
\hline Bushehr & 5.86 & 1.14 & 3.74 & $\begin{array}{l}\text { Kohgiluyeh } \\
\text { and Boyer- } \\
\text { Ahmad }\end{array}$ & 2.73 & 0.53 & 1.74 \\
\hline Tehran & 197.97 & 38.71 & 126.35 & Golestan & 11.24 & 2.19 & 7.17 \\
\hline $\begin{array}{l}\text { ChaharMahaal } \\
\text { and Bakhtiari }\end{array}$ & 18.12 & 3.54 & 11.56 & Gilan & 2.32 & 0.45 & 1.48 \\
\hline $\begin{array}{l}\text { Khorasan, } \\
\text { South }\end{array}$ & 7.07 & 1.38 & 4.51 & Lorestan & 6.09 & 1.19 & 3.88 \\
\hline $\begin{array}{c}\text { Khorasan, } \\
\text { Razavi }\end{array}$ & 69.36 & 13.56 & 44.27 & Mazandaran & 13.33 & 2.60 & 8.51 \\
\hline $\begin{array}{l}\text { Khorasan, } \\
\text { North }\end{array}$ & 4.69 & 0.91 & 2.99 & Markazi & 36.99 & 7.23 & 23.60 \\
\hline Khuzestan & 14.28 & 2.79 & 9.11 & Hormozgan & 0.78 & 0.15 & 0.49 \\
\hline Zanjan & 10.48 & 2.05 & 6.69 & Hamadan & 10.79 & 2.11 & 6.88 \\
\hline Semnan & 35.50 & 6.94 & 22.66 & Yazd & 37.47 & 7.32 & 23.91 \\
\hline $\begin{array}{l}\text { Sistan and } \\
\text { Baluchestan }\end{array}$ & 2.53 & 0.49 & 1.61 & Iran & 886.57 & 173.36 & 565.85 \\
\hline
\end{tabular}

The manure and biogas characteristics for calculating the potential of biogas production from the livestock manure based on Plume et al. (2012) such as dry matter content, organic matter content in dry matter, biogas output from manure and heat energy of biogas are shown in Table 9 .

Table 9. Manure and biogas characteristics in different groups of livestock based on Plume et al. (2012)

\begin{tabular}{c|c}
\hline Groups of livestock & Dairy cows, heifers, calves, cattle \\
\hline Dry matter content & 0.18 \\
Organic matter content in dry matter & 0.86 \\
Biogas output from manure $\left(\mathrm{m}^{3} \mathrm{t}^{-1}\right)$ & 300 \\
Heat energy of biogas $\left(\mathrm{kWh} \mathrm{m}^{-3}\right)$ & 5.8 \\
\hline
\end{tabular}

The biogas production potential of livestock manure from the different groups of livestock in Iran was calculated based on Plume et al. (2012) using Equation 5 and is presented in Table 10. 


$$
-6511-
$$

Table 10. The estimated values of biogas production of different provinces in Iran in 2016 based on Plume et al. (2012)

\begin{tabular}{|c|c|c|c|c|c|c|}
\hline \multicolumn{7}{|c|}{ Biogas production (million $\mathrm{m}^{3} \mathrm{yr}^{-1}$ ) } \\
\hline \multirow{2}{*}{ Province } & \multicolumn{2}{|c|}{ Dairy cows } & \multirow{2}{*}{ Heifers } & \multirow{2}{*}{ Calves } & \multirow{2}{*}{ Cattle } & \multirow{2}{*}{ Total } \\
\hline & Lactating cow & Dry cow & & & & \\
\hline Azerbaijan, West & 9.28 & 1.64 & 2.09 & 0.83 & 2.11 & 15.96 \\
\hline Azerbaijan, East & 3.35 & 0.90 & 0.78 & 0.51 & 0.79 & 6.35 \\
\hline Ardabil & 5.92 & 1.31 & 1.49 & 1.02 & 1.15 & 10.92 \\
\hline Isfahan & 103.03 & 13.81 & 15.75 & 5.79 & 3.55 & 141.95 \\
\hline Alborz & 28.20 & 5.01 & 5.35 & 2.01 & 1.81 & 42.40 \\
\hline Ilam & 1.29 & 0.86 & 0.23 & 0.20 & 0.40 & 3.00 \\
\hline Bushehr & 0.19 & 0.55 & 0.24 & 0.78 & 0.20 & 1.97 \\
\hline Tehran & 73.43 & 20.07 & 18.39 & 16.11 & 5.22 & 133.24 \\
\hline ChaharMahaal and Bakhtiari & 10.82 & 3.21 & 2.14 & 0.81 & 0.56 & 17.56 \\
\hline Khorasan, South & 3.36 & 1.03 & 0.83 & 0.37 & 0.45 & 6.06 \\
\hline Khorasan, Razavi & 45.52 & 7.89 & 8.29 & 3.27 & 3.56 & 68.55 \\
\hline Khorasan, North & 2.23 & 0.44 & 0.45 & 0.32 & 0.36 & 3.82 \\
\hline Khuzestan & 2.88 & 1.02 & 0.59 & 1.50 & 1.91 & 7.92 \\
\hline Zanjan & 7.22 & 1.13 & 1.51 & 0.41 & 0.81 & 11.10 \\
\hline Semnan & 13.33 & 3.01 & 2.74 & 1.70 & 7.11 & 27.91 \\
\hline Sistan and Baluchestan & 0.37 & 0.30 & 0.10 & 0.19 & 0.60 & 1.57 \\
\hline Fars & 34.03 & 6.44 & 8.20 & 3.56 & 4.79 & 57.05 \\
\hline Qazvin & 28.11 & 3.82 & 4.96 & 2.62 & 2.40 & 41.93 \\
\hline Qom & 11.54 & 1.69 & 2.00 & 4.86 & 3.66 & 23.77 \\
\hline Kurdistan & 1.06 & 0.13 & 0.19 & 0.16 & 0.12 & 1.68 \\
\hline Kerman & 9.13 & 2.78 & 2.44 & 0.96 & 1.81 & 17.15 \\
\hline Kermanshah & 7.41 & 1.17 & 1.29 & 0.41 & 0.90 & 11.20 \\
\hline Kohgiluyeh and Boyer-Ahmad & 1.57 & 0.20 & 0.33 & 0.21 & 0.01 & 2.35 \\
\hline Golestan & 5.49 & 1.68 & 1.68 & 0.55 & 0.27 & 9.69 \\
\hline Gilan & 0.95 & 0.30 & 0.30 & 0.11 & 0.14 & 1.82 \\
\hline Lorestan & 2.24 & 0.52 & 0.57 & 0.52 & 0.10 & 3.97 \\
\hline Mazandaran & 8.40 & 1.69 & 1.52 & 0.60 & 1.08 & 13.32 \\
\hline Markazi & 7.29 & 1.74 & 1.59 & 4.71 & 2.09 & 17.44 \\
\hline Hormozgan & 0.09 & 0.06 & 0.02 & 0.04 & 0.27 & 0.49 \\
\hline Hamadan & 6.55 & 1.62 & 0.94 & 0.67 & 0.33 & 10.13 \\
\hline Yazd & 19.63 & 4.65 & 5.00 & 1.73 & 1.95 & 32.981 \\
\hline Iran & 454.04 & 90.80 & 92.15 & 57.67 & 50.68 & 745.37 \\
\hline
\end{tabular}

Calculation of the potential of electricity generation from the livestock manure in Iran

The potential of electricity generation from the biogas obtainable from livestock manure calculated based on Abdeshahian, Boysan, Zareei and Plume methods was estimated for all provinces of Iran according to Equations 3 and 4 and is presented in Table 11. 
Table 11. The estimated values of the potential of electricity generation from livestock manure based on different methods in all provinces of Iran in 2016

\begin{tabular}{|c|c|c|c|c|c|c|c|c|c|}
\hline \multicolumn{10}{|c|}{ 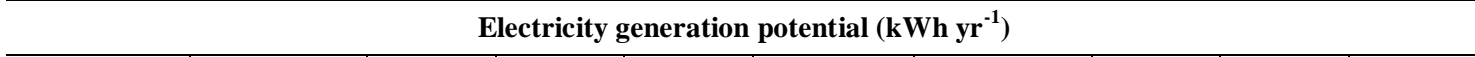 } \\
\hline Province & Abdeshahian & Boysan & Zareei & Plume & Province & Abdeshahian & Boysan & Zareei & Plume \\
\hline $\begin{array}{l}\text { Azerbaijan, } \\
\text { West }\end{array}$ & $3.14 \mathrm{E}+07$ & $6.15 \mathrm{E}+06$ & $2.01 \mathrm{E}+07$ & $2.87 \mathrm{E}+07$ & Fars & $1.17 \mathrm{E}+08$ & $2.28 \mathrm{E}+07$ & $7.44 \mathrm{E}+07$ & $1.03 \mathrm{E}+08$ \\
\hline $\begin{array}{l}\text { Azerbaijan, } \\
\text { East }\end{array}$ & $1.46 \mathrm{E}+07$ & $2.86 \mathrm{E}+06$ & $9.35 \mathrm{E}+06$ & $1.14 \mathrm{E}+07$ & Qazvin & $8.05 E+07$ & $1.57 \mathrm{E}+07$ & $5.14 \mathrm{E}+07$ & $7.55 \mathrm{E}+07$ \\
\hline Ardabil & $2.77 \mathrm{E}+07$ & $5.41 \mathrm{E}+06$ & $1.77 \mathrm{E}+07$ & $1.97 \mathrm{E}+07$ & Qom & 7.19E+07 & $1.41 \mathrm{E}+07$ & $4.59 \mathrm{E}+07$ & $4.28 \mathrm{E}+07$ \\
\hline Isfahan & $2.29 \mathrm{E}+08$ & $4.49 \mathrm{E}+07$ & $1.46 \mathrm{E}+08$ & $2.56 \mathrm{E}+08$ & Kurdistan & $3.80 \mathrm{E}+06$ & $7.43 \mathrm{E}+05$ & $2.43 \mathrm{E}+06$ & $3.02 \mathrm{E}+06$ \\
\hline Alborz & $8.02 \mathrm{E}+07$ & $1.57 \mathrm{E}+07$ & $5.12 \mathrm{E}+07$ & $7.63 \mathrm{E}+07$ & Kerman & $3.68 \mathrm{E}+07$ & 7.20E+06 & $2.35 \mathrm{E}+07$ & $3.09 \mathrm{E}+07$ \\
\hline Ilam & $6.82 \mathrm{E}+06$ & $1.33 \mathrm{E}+06$ & $4.35 \mathrm{E}+06$ & $5.41 \mathrm{E}+06$ & Kermanshah & $1.83 \mathrm{E}+07$ & $3.59 \mathrm{E}+06$ & $1.17 \mathrm{E}+07$ & $2.02 \mathrm{E}+07$ \\
\hline Bushehr & $1.06 \mathrm{E}+07$ & $2.06 \mathrm{E}+06$ & $6.74 \mathrm{E}+06$ & $3.55 \mathrm{E}+06$ & $\begin{array}{c}\text { Kohgiluyeh } \\
\text { and Boyer- } \\
\text { Ahmad }\end{array}$ & $4.92 \mathrm{E}+06$ & $9.61 \mathrm{E}+05$ & $3.14 \mathrm{E}+06$ & $4.24 \mathrm{E}+06$ \\
\hline Tehran & $3.56 \mathrm{E}+08$ & $6.97 \mathrm{E}+07$ & $2.27 \mathrm{E}+08$ & $2.40 \mathrm{E}+08$ & Golestan & $2.02 \mathrm{E}+07$ & $3.96 \mathrm{E}+06$ & $1.29 \mathrm{E}+07$ & $1.74 \mathrm{E}+07$ \\
\hline $\begin{array}{l}\text { ChaharMahaal } \\
\text { and Bakhtiari }\end{array}$ & $3.26 \mathrm{E}+07$ & $6.38 \mathrm{E}+06$ & $2.08 \mathrm{E}+07$ & $3.16 \mathrm{E}+07$ & Gilan & $4.18 \mathrm{E}+06$ & $8.17 \mathrm{E}+05$ & $2.67 \mathrm{E}+06$ & $3.28 \mathrm{E}+06$ \\
\hline $\begin{array}{l}\text { Khorasan, } \\
\text { South }\end{array}$ & $1.27 \mathrm{E}+07$ & $2.49 \mathrm{E}+06$ & $8.13 \mathrm{E}+06$ & $1.09 \mathrm{E}+07$ & Lorestan & $1.10 \mathrm{E}+07$ & $2.14 \mathrm{E}+06$ & $7.00 \mathrm{E}+06$ & $7.16 \mathrm{E}+06$ \\
\hline $\begin{array}{l}\text { Khorasan, } \\
\text { Razavi }\end{array}$ & $1.25 \mathrm{E}+08$ & $2.44 \mathrm{E}+07$ & 7.97E+07 & $1.23 \mathrm{E}+08$ & Mazandaran & $2.40 \mathrm{E}+07$ & $4.69 \mathrm{E}+06$ & $1.53 \mathrm{E}+07$ & $2.40 \mathrm{E}+07$ \\
\hline $\begin{array}{l}\text { Khorasan, } \\
\text { North }\end{array}$ & $8.46 \mathrm{E}+06$ & $1.65 \mathrm{E}+06$ & $5.40 \mathrm{E}+06$ & $6.88 \mathrm{E}+06$ & Markazi & $6.66 \mathrm{E}+07$ & $1.30 \mathrm{E}+07$ & $4.25 \mathrm{E}+07$ & $3.14 \mathrm{E}+07$ \\
\hline Khuzestan & $2.57 \mathrm{E}+07$ & $5.03 \mathrm{E}+06$ & $1.64 \mathrm{E}+07$ & $1.43 \mathrm{E}+07$ & Hormozgan & $1.41 \mathrm{E}+06$ & $2.75 \mathrm{E}+05$ & $8.99 \mathrm{E}+05$ & $8.93 E+05$ \\
\hline Zanjan & $1.89 \mathrm{E}+07$ & $3.69 \mathrm{E}+06$ & $1.20 \mathrm{E}+07$ & $2.00 \mathrm{E}+07$ & Hamadan & $1.94 \mathrm{E}+07$ & $3.80 \mathrm{E}+06$ & $1.24 \mathrm{E}+07$ & $1.82 \mathrm{E}+07$ \\
\hline Semnan & $6.39 \mathrm{E}+07$ & $1.25 \mathrm{E}+07$ & $4.08 \mathrm{E}+07$ & $5.03 \mathrm{E}+07$ & Yazd & $6.74 \mathrm{E}+07$ & $1.32 \mathrm{E}+07$ & $4.30 \mathrm{E}+07$ & $5.94 \mathrm{E}+07$ \\
\hline $\begin{array}{l}\text { Sistan and } \\
\text { Baluchestan }\end{array}$ & $4.56 \mathrm{E}+06$ & $8.92 \mathrm{E}+05$ & $2.91 \mathrm{E}+06$ & $2.84 \mathrm{E}+06$ & Iran & $1.60 \mathrm{E}+09$ & $3.12 \mathrm{E}+08$ & $1.02 \mathrm{E}+09$ & $1.34 \mathrm{E}+09$ \\
\hline
\end{tabular}

\section{Calculation of the potential of energy of biogas from the livestock manure in Iran}

The potential of energy of biogas obtainable from manure biomass calculated based on Abdeshahina, Boysan, Zareei and Plume Methods in all provinces of Iran was estimated using Equation 6 and is presented in Table 12.

Table 12. The estimated values of the biogas energy obtainable from manure biomass based on different methods in all provinces of Iran in 2016

\begin{tabular}{c|c|c|c|c|c|c|c|c|c}
\hline \multicolumn{7}{c}{ The potential of biogas energy obtainable from manure $(\mathbf{k W h})$} \\
\hline $\begin{array}{c}\text { Province } \\
\text { Azerbaijan, } \\
\text { West } \\
\begin{array}{c}\text { Azerbaijan, } \\
\text { East }\end{array}\end{array}$ & $1.01 \mathrm{E}+08$ & $1.98 \mathrm{E}+07$ & $6.47 \mathrm{E}+07$ & $9.26 \mathrm{E}+07$ & Fars & $3.76 \mathrm{E}+08$ & $7.34 \mathrm{E}+07$ & $2.40 \mathrm{E}+08$ & $3.31 \mathrm{E}+08$ \\
Ardabil & $8.72 \mathrm{E}+07$ & $9.23 \mathrm{E}+06$ & $3.01 \mathrm{E}+07$ & $3.69 \mathrm{E}+07$ & Qazvin & $2.59 \mathrm{E}+08$ & $5.07 \mathrm{E}+07$ & $1.65 \mathrm{E}+08$ & $2.43 \mathrm{E}+08$ \\
Isfahan & $7.39 \mathrm{E}+08$ & $1.45 \mathrm{E}+08$ & $4.72 \mathrm{E}+08$ & $8.23 \mathrm{E}+08$ & Kurdistan & $1.22 \mathrm{E}+07$ & $2.39 \mathrm{E}+06$ & $7.77 \mathrm{E}+06$ & $9.74 \mathrm{E}+06$ \\
Alborz & $2.58 \mathrm{E}+08$ & $5.05 \mathrm{E}+07$ & $1.65 \mathrm{E}+08$ & $2.46 \mathrm{E}+08$ & Kerman & $1.19 \mathrm{E}+08$ & $2.32 \mathrm{E}+07$ & $7.56 \mathrm{E}+07$ & $9.95 \mathrm{E}+07$ \\
Ilam & $2.20 \mathrm{E}+07$ & $4.30 \mathrm{E}+06$ & $1.40 \mathrm{E}+07$ & $1.74 \mathrm{E}+07$ & Kermanshah & $5.91 \mathrm{E}+07$ & $1.16 \mathrm{E}+07$ & $3.77 \mathrm{E}+07$ & $6.50 \mathrm{E}+07$ \\
\hline
\end{tabular}


Table 12 (continued). The estimated values of the biogas energy obtainable from manure biomass based on different methods in all provinces of Iran in 2016

\begin{tabular}{|c|c|c|c|c|c|c|c|c|c|}
\hline \multicolumn{10}{|c|}{ The potential of biogas energy obtainable from manure $(\mathrm{kWh})$} \\
\hline Province & Abdeshahian & Boysan & Zareei & Plume & Province & Abdeshahian & Boysan & Zareei & Plume \\
\hline Bushehr & $3.40 \mathrm{E}+07$ & $6.65 \mathrm{E}+06$ & $2.17 \mathrm{E}+07$ & $1.14 \mathrm{E}+07$ & $\begin{array}{l}\text { Kohgiluyeh } \\
\text { and Boyer- } \\
\text { Ahmad }\end{array}$ & $1.58 \mathrm{E}+07$ & $3.10 \mathrm{E}+06$ & $1.01 \mathrm{E}+07$ & $1.37 \mathrm{E}+07$ \\
\hline Tehran & $1.15 \mathrm{E}+09$ & $2.25 \mathrm{E}+08$ & 7.33E+08 & $7.73 \mathrm{E}+08$ & Golestan & $6.52 \mathrm{E}+07$ & $1.28 \mathrm{E}+07$ & $4.16 \mathrm{E}+07$ & $5.62 \mathrm{E}+07$ \\
\hline $\begin{array}{l}\text { ChaharMahaal } \\
\text { and Bakhtiari }\end{array}$ & $1.05 \mathrm{E}+08$ & $2.06 \mathrm{E}+07$ & $6.70 \mathrm{E}+07$ & $1.02 \mathrm{E}+08$ & Gilan & $1.35 \mathrm{E}+07$ & $2.63 \mathrm{E}+06$ & $8.58 \mathrm{E}+06$ & $1.06 \mathrm{E}+07$ \\
\hline $\begin{array}{l}\text { Khorasan, } \\
\text { South }\end{array}$ & $4.11 \mathrm{E}+07$ & $8.03 E+06$ & $2.62 \mathrm{E}+07$ & $3.52 \mathrm{E}+07$ & Lorestan & $3.53 \mathrm{E}+07$ & $6.91 \mathrm{E}+06$ & $2.25 \mathrm{E}+07$ & $2.31 \mathrm{E}+07$ \\
\hline $\begin{array}{c}\text { Khorasan, } \\
\text { Razavi }\end{array}$ & $4.02 \mathrm{E}+08$ & $7.87 \mathrm{E}+07$ & $2.57 \mathrm{E}+08$ & $3.98 \mathrm{E}+08$ & Mazandaran & $7.73 \mathrm{E}+07$ & $1.51 \mathrm{E}+07$ & $4.94 \mathrm{E}+07$ & $7.73 \mathrm{E}+07$ \\
\hline $\begin{array}{l}\text { Khorasan, } \\
\text { North }\end{array}$ & $2.72 \mathrm{E}+07$ & $5.33 \mathrm{E}+06$ & $1.73 \mathrm{E}+07$ & $2.22 \mathrm{E}+07$ & Markazi & $2.15 \mathrm{E}+08$ & $4.20 \mathrm{E}+07$ & $1.37 \mathrm{E}+08$ & $1.01 \mathrm{E}+08$ \\
\hline Khuzestan & $8.28 \mathrm{E}+07$ & $1.62 \mathrm{E}+07$ & $5.28 \mathrm{E}+07$ & $4.60 \mathrm{E}+07$ & Hormozgan & $4.54 \mathrm{E}+06$ & $8.87 \mathrm{E}+05$ & $2.84 \mathrm{E}+06$ & $2.88 \mathrm{E}+06$ \\
\hline Zanjan & $6.08 \mathrm{E}+07$ & $1.19 \mathrm{E}+07$ & $3.88 \mathrm{E}+07$ & $6.44 \mathrm{E}+07$ & Hamadan & $6.26 \mathrm{E}+07$ & $1.22 \mathrm{E}+07$ & $3.99 \mathrm{E}+07$ & $5.88 \mathrm{E}+07$ \\
\hline Semnan & $2.06 \mathrm{E}+08$ & $4.03 \mathrm{E}+07$ & $1.31 \mathrm{E}+08$ & $1.62 \mathrm{E}+08$ & Yazd & $2.17 \mathrm{E}+08$ & $4.25 \mathrm{E}+07$ & $1.39 \mathrm{E}+08$ & $1.91 \mathrm{E}+08$ \\
\hline $\begin{array}{c}\text { Sistan and } \\
\text { Baluchestan }\end{array}$ & $1.47 \mathrm{E}+07$ & $2.87 \mathrm{E}+06$ & $9.34 \mathrm{E}+06$ & $9.16 \mathrm{E}+06$ & Iran & $5.14 \mathrm{E}+09$ & $1.01 \mathrm{E}+09$ & $3.28 \mathrm{E}+09$ & $4.32 \mathrm{E}+09$ \\
\hline
\end{tabular}

\section{Calculation of methane $\left(\mathrm{CH}_{4}\right)$ content of biogas from manure livestock in Iran}

The chemical composition of the livestock manure which is required to calculate the biochemical methane potential based on $\mathrm{Cu}$ et al. (2015) including dry matter, volatile solid, protein, lipid, cellulose and lignin are shown in Table 13.

Table 13. Chemical composition of livestock manure based on Cu et al. (2015)

\begin{tabular}{c|c}
\hline Substrates & Animal manure group \\
\hline Dry matter & 10.94 \\
Volatile solid $^{1}$ & 73.01 \\
Protein $^{1}$ & 7.55 \\
Lipid $^{1}$ & 3.63 \\
Cellulose $^{1}$ & 17.59 \\
Lignin $^{1}$ & 10.41 \\
\hline
\end{tabular}

\footnotetext{
${ }^{1} \%$ in dry matter
}

The BMP value based on chemical composition of the substrate was calculated according to chemical composition of the livestock manure (Table 13) and equations to predict BMP (Table 3) and is presented in Table 14.

Table 14. The estimated values of BMP based on chemical composition of the substrate

\begin{tabular}{c|c}
\hline Variables & BMP \\
\hline Lipid & 184.95 \\
Lipid, lignin & 243.67 \\
Lipid, lignin, dry matter & 232.31 \\
Lipid, lignin, cellulose & 242.34 \\
\hline
\end{tabular}




$$
-6514-
$$

The methane yield was calculated in different provinces of Iran according to the BMP value $\left(\beta_{a}\right.$ in the extended Hashimoto equation by Rennuit and Sommer (2013)) from different types of substrate based on chemical composition (Table 14) and is presented in Tables 15-18.

Table 15. The estimated values of methane yield ( $\mathrm{NL} \mathrm{CH}_{4}$ digester ${ }^{-1}$ day $\left.{ }^{-1}\right)$ based on the BMP value of specific substrate (lipid)

\begin{tabular}{|c|c|c|c|c|c|c|c|}
\hline Province & $\begin{array}{l}\text { Methane } \\
\text { yield }\end{array}$ & Province & $\begin{array}{l}\text { Methane } \\
\text { yield }\end{array}$ & Province & $\begin{array}{l}\text { Methane } \\
\text { yield }\end{array}$ & Province & $\begin{array}{c}\text { Methane } \\
\text { yield }\end{array}$ \\
\hline $\begin{array}{l}\text { Azerbaijan, } \\
\text { West }\end{array}$ & $5.64 \mathrm{E}+08$ & $\begin{array}{l}\text { ChaharMahaal } \\
\text { and Bakhtiari }\end{array}$ & $3.13 \mathrm{E}+08$ & Fars & $3.19 \mathrm{E}+09$ & Gilan & $7.90 \mathrm{E}+07$ \\
\hline $\begin{array}{c}\text { Azerbaijan, } \\
\text { East }\end{array}$ & $1.72 \mathrm{E}+08$ & $\begin{array}{l}\text { Khorasan, } \\
\text { South }\end{array}$ & $2.89 \mathrm{E}+08$ & Qazvin & $1.45 \mathrm{E}+09$ & Lorestan & $2.60 \mathrm{E}+08$ \\
\hline Ardabil & $1.41 \mathrm{E}+08$ & $\begin{array}{c}\text { Khorasan, } \\
\text { Razavi }\end{array}$ & $2.65 \mathrm{E}+09$ & Qom & $2.20 \mathrm{E}+09$ & Mazandaran & $5.53 \mathrm{E}+08$ \\
\hline Isfahan & $5.94 \mathrm{E}+09$ & $\begin{array}{l}\text { Khorasan, } \\
\text { North }\end{array}$ & $1.33 \mathrm{E}+08$ & Kurdistan & $7.02 \mathrm{E}+07$ & Markazi & $1.29 \mathrm{E}+09$ \\
\hline Alborz & $1.70 \mathrm{E}+09$ & Khuzestan & $1.24 \mathrm{E}+09$ & Kerman & $8.38 \mathrm{E}+08$ & Hormozgan & $7.31 \mathrm{E}+07$ \\
\hline Ilam & $1.52 \mathrm{E}+08$ & Zanjan & $2.33 \mathrm{E}+08$ & Kermanshah & $3.73 \mathrm{E}+08$ & Hamadan & $2.67 \mathrm{E}+08$ \\
\hline Bushehr & $4.98 \mathrm{E}+08$ & Semnan & $2.00 \mathrm{E}+09$ & $\begin{array}{c}\text { Kohgiluyeh } \\
\text { and Boyer- } \\
\text { Ahmad }\end{array}$ & $7.68 \mathrm{E}+07$ & Yazd & $2.28 \mathrm{E}+09$ \\
\hline Tehran & $1.05 \mathrm{E}+10$ & $\begin{array}{l}\text { Sistan and } \\
\text { Baluchestan }\end{array}$ & $1.32 \mathrm{E}+08$ & Golestan & $4.71 \mathrm{E}+08$ & Iran & $4.01 \mathrm{E}+10$ \\
\hline
\end{tabular}

Table 16. The estimated values of methane yield $\left(\mathrm{NL} \mathrm{CH}_{4}\right.$ digester ${ }^{-1}$ day $\left.{ }^{-1}\right)$ based on the BMP value of specific substrate (lipid and lignin)

\begin{tabular}{|c|c|c|c|c|c|c|c|}
\hline Province & $\begin{array}{l}\text { Methane } \\
\text { yield }\end{array}$ & Province & $\begin{array}{l}\text { Methane } \\
\text { yield }\end{array}$ & Province & $\begin{array}{l}\text { Methane } \\
\text { yield }\end{array}$ & Province & $\begin{array}{l}\text { Methane } \\
\text { yield }\end{array}$ \\
\hline $\begin{array}{l}\text { Azerbaijan, } \\
\text { West }\end{array}$ & $7.42 \mathrm{E}+08$ & $\begin{array}{l}\text { ChaharMahaal } \\
\text { and Bakhtiari }\end{array}$ & $4.12 \mathrm{E}+08$ & Fars & 4.2E+09 & Gilan & $1.04 \mathrm{E}+08$ \\
\hline $\begin{array}{c}\text { Azerbaijan, } \\
\text { East }\end{array}$ & $2.26 \mathrm{E}+08$ & $\begin{array}{l}\text { Khorasan, } \\
\text { South }\end{array}$ & $3.81 \mathrm{E}+08$ & Qazvin & $1.9 \mathrm{E}+09$ & Lorestan & $3.43 \mathrm{E}+08$ \\
\hline Ardabil & $1.85 \mathrm{E}+08$ & $\begin{array}{c}\text { Khorasan, } \\
\text { Razavi }\end{array}$ & $3.49 \mathrm{E}+09$ & Qom & $2.9 \mathrm{E}+09$ & Mazandaran & $7.27 \mathrm{E}+08$ \\
\hline Isfahan & $7.82 \mathrm{E}+09$ & $\begin{array}{c}\text { Khorasan, } \\
\text { North }\end{array}$ & $1.75 \mathrm{E}+08$ & Kurdistan & 92446950 & Markazi & $1.7 \mathrm{E}+09$ \\
\hline Alborz & $2.23 \mathrm{E}+09$ & Khuzestan & $1.64 \mathrm{E}+09$ & Kerman & $1.1 \mathrm{E}+09$ & Hormozgan & 97949190 \\
\hline Ilam & $1.99 \mathrm{E}+08$ & Zanjan & $3.07 \mathrm{E}+08$ & Kermanshah & $4.9 \mathrm{E}+08$ & Hamadan & $3.51 \mathrm{E}+08$ \\
\hline Bushehr & $6.57 \mathrm{E}+08$ & Semnan & $2.63 \mathrm{E}+09$ & $\begin{array}{c}\text { Kohgiluyeh } \\
\text { and Boyer- } \\
\text { Ahmad }\end{array}$ & $1.02 \mathrm{E}+08$ & Yazd & $3 \mathrm{E}+09$ \\
\hline Tehran & $1.38 \mathrm{E}+10$ & $\begin{array}{l}\text { Sistan and } \\
\text { Baluchestan }\end{array}$ & $1.74 \mathrm{E}+08$ & Golestan & $6.2 \mathrm{E}+08$ & Iran & $5.27 \mathrm{E}+10$ \\
\hline
\end{tabular}




$$
-6515-
$$

Table 17. The estimated values of methane yield ( $\mathrm{NL} \mathrm{CH}_{4}$ digester ${ }^{-1}$ day $\left.{ }^{-1}\right)$ based on the BMP value of specific substrate (lipid, lignin and dry matter)

\begin{tabular}{|c|c|c|c|c|c|c|c|}
\hline Province & $\begin{array}{l}\text { Methane } \\
\text { yield }\end{array}$ & Province & $\begin{array}{l}\text { Methane } \\
\text { yield }\end{array}$ & Province & $\begin{array}{c}\text { Methane } \\
\text { yield }\end{array}$ & Province & $\begin{array}{c}\text { Methane } \\
\text { yield }\end{array}$ \\
\hline $\begin{array}{l}\text { Azerbaijan, } \\
\text { West }\end{array}$ & $7.08 \mathrm{E}+08$ & $\begin{array}{l}\text { ChaharMahaal } \\
\text { and Bakhtiari }\end{array}$ & $3.93 \mathrm{E}+08$ & Fars & $4.01 \mathrm{E}+09$ & Gilan & $9.90 \mathrm{E}+07$ \\
\hline $\begin{array}{l}\text { Azerbaijan, } \\
\text { East }\end{array}$ & $2.15 \mathrm{E}+08$ & $\begin{array}{l}\text { Khorasan, } \\
\text { South }\end{array}$ & $3.63 \mathrm{E}+08$ & Qazvin & $1.81 \mathrm{E}+09$ & Lorestan & $3.27 \mathrm{E}+08$ \\
\hline Ardabil & $1.77 \mathrm{E}+08$ & $\begin{array}{l}\text { Khorasan, } \\
\text { Razavi }\end{array}$ & $3.33 \mathrm{E}+09$ & Qom & $2.77 \mathrm{E}+09$ & Mazandaran & $6.93 \mathrm{E}+08$ \\
\hline Isfahan & $7.46 \mathrm{E}+09$ & $\begin{array}{l}\text { Khorasan, } \\
\text { North }\end{array}$ & $1.66 \mathrm{E}+08$ & Kurdistan & $8.81 \mathrm{E}+07$ & Markazi & $1.62 \mathrm{E}+09$ \\
\hline Alborz & $2.13 \mathrm{E}+09$ & Khuzestan & $1.56 \mathrm{E}+09$ & Kerman & $1.05 \mathrm{E}+09$ & Hormozgan & $9.17 \mathrm{E}+07$ \\
\hline Ilam & $1.9 \mathrm{E}+08$ & Zanjan & $2.93 \mathrm{E}+08$ & Kermanshah & $4.68 \mathrm{E}+08$ & Hamadan & $3.34 \mathrm{E}+08$ \\
\hline Bushehr & $6.26 \mathrm{E}+08$ & Semnan & $2.5 \mathrm{E}+09$ & $\begin{array}{c}\text { Kohgiluyeh } \\
\text { and Boyer- } \\
\text { Ahmad }\end{array}$ & $9.71 \mathrm{E}+07$ & Yazd & $2.86 \mathrm{E}+09$ \\
\hline Tehran & $1.31 \mathrm{E}+10$ & $\begin{array}{c}\text { Sistan and } \\
\text { Baluchestan }\end{array}$ & $1.66 \mathrm{E}+08$ & Golestan & $5.91 \mathrm{E}+08$ & Iran & $5.03 \mathrm{E}+10$ \\
\hline
\end{tabular}

Table 18. The estimated values of methane yield $\left(\mathrm{NL} \mathrm{CH}_{4}\right.$ digester $^{-1}$ day $\left.{ }^{-1}\right)$ based on the BMP value of specific substrate (lipid, lignin and cellulose)

\begin{tabular}{|c|c|c|c|c|c|c|c|}
\hline Province & $\begin{array}{l}\text { Methane } \\
\text { yield }\end{array}$ & Province & $\begin{array}{l}\text { Methane } \\
\text { yield }\end{array}$ & Province & $\begin{array}{l}\text { Methane } \\
\text { yield }\end{array}$ & Province & $\begin{array}{l}\text { Methane } \\
\text { yield }\end{array}$ \\
\hline $\begin{array}{l}\text { Azerbaijan, } \\
\text { West }\end{array}$ & $7.38 \mathrm{E}+08$ & $\begin{array}{l}\text { ChaharMahaal } \\
\text { and Bakhtiari }\end{array}$ & $4.09 \mathrm{E}+08$ & Fars & $4.18 \mathrm{E}+09$ & Gilan & $1.03 \mathrm{E}+08$ \\
\hline $\begin{array}{c}\text { Azerbaijan, } \\
\text { East }\end{array}$ & $2.25 \mathrm{E}+08$ & $\begin{array}{l}\text { Khorasan, } \\
\text { South }\end{array}$ & $3.79 \mathrm{E}+08$ & Qazvin & $1.89 \mathrm{E}+09$ & Lorestan & $3.41 \mathrm{E}+08$ \\
\hline Ardabil & $1.84 \mathrm{E}+08$ & $\begin{array}{c}\text { Khorasan, } \\
\text { Razavi }\end{array}$ & $3.47 \mathrm{E}+09$ & Qom & $2.89 \mathrm{E}+09$ & Mazandaran & $7.23 \mathrm{E}+08$ \\
\hline Isfahan & $7.78 \mathrm{E}+09$ & $\begin{array}{l}\text { Khorasan, } \\
\text { North }\end{array}$ & $1.74 \mathrm{E}+08$ & Kurdistan & 91942230 & Markazi & $1.69 \mathrm{E}+09$ \\
\hline Alborz & $2.22 \mathrm{E}+09$ & Khuzestan & $1.63 \mathrm{E}+09$ & Kerman & $1.1 \mathrm{E}+09$ & Hormozgan & 95695200 \\
\hline Ilam & $1.98 \mathrm{E}+08$ & Zanjan & $3.05 \mathrm{E}+08$ & Kermanshah & $4.88 \mathrm{E}+08$ & Hamadan & $3.49 \mathrm{E}+08$ \\
\hline Bushehr & $6.53 \mathrm{E}+08$ & Semnan & $2.61 \mathrm{E}+09$ & $\begin{array}{c}\text { Kohgiluyeh } \\
\text { and Boyer- } \\
\text { Ahmad }\end{array}$ & $1.01 \mathrm{E}+08$ & Yazd & $2.98 \mathrm{E}+09$ \\
\hline Tehran & $1.37 \mathrm{E}+10$ & $\begin{array}{c}\text { Sistan and } \\
\text { Baluchestan }\end{array}$ & $1.73 \mathrm{E}+08$ & Golestan & $6.16 \mathrm{E}+08$ & Iran & $5.25 \mathrm{E}+10$ \\
\hline
\end{tabular}

The potential of livestock manure production, biogas, electricity and energy obtainable from livestock manure and methane yield of biogas were calculated for all provinces in Iran in order to compare the results between provinces and determine which of them has the least and most potential. The amount of livestock manure production in different provinces in Iran based on Abdeshahian, Boysan and Zareei methods (Table 4), shows that the higher amount of the manure was produced in Tehran while the lowest amount of the manure is related to Hormozgan and it is because of this 


$$
-6516-
$$

that Tehran and Hormozgan have the highest and the lowest number of livestock, respectively, compared to that other provinces of Iran. On the other hand, the amount of livestock manure based on Plume method (Table 7) shows that the higher amount of the manure was produced in Isfahan. This is due to considering the different manure production for each group of livestock (dairy cows, heifers, calves and cattle) in this method. The lowest amount of manure production still belongs to Hormozgan.

Figure 2 shows the potential of livestock manure production in Iran according to 4 different methods. It is shown that the higher amount of the manure was produced in Plume method with the annual manure production of 16.05 million ton followed by Abdeshahian, Boysan and Zareei methods with a manure production of 11.82, 5.25 and 2.1 tons, respectively. As can be seen, the amount of livestock manure produced using Zareei method was much less compared to that from Plume, Abdeshahian and Boysan methods. This is due to the fact that the lowest coefficient of manure production was considered in this method (the ratio of the annual livestock manure to livestock weight $=2.6$ ).

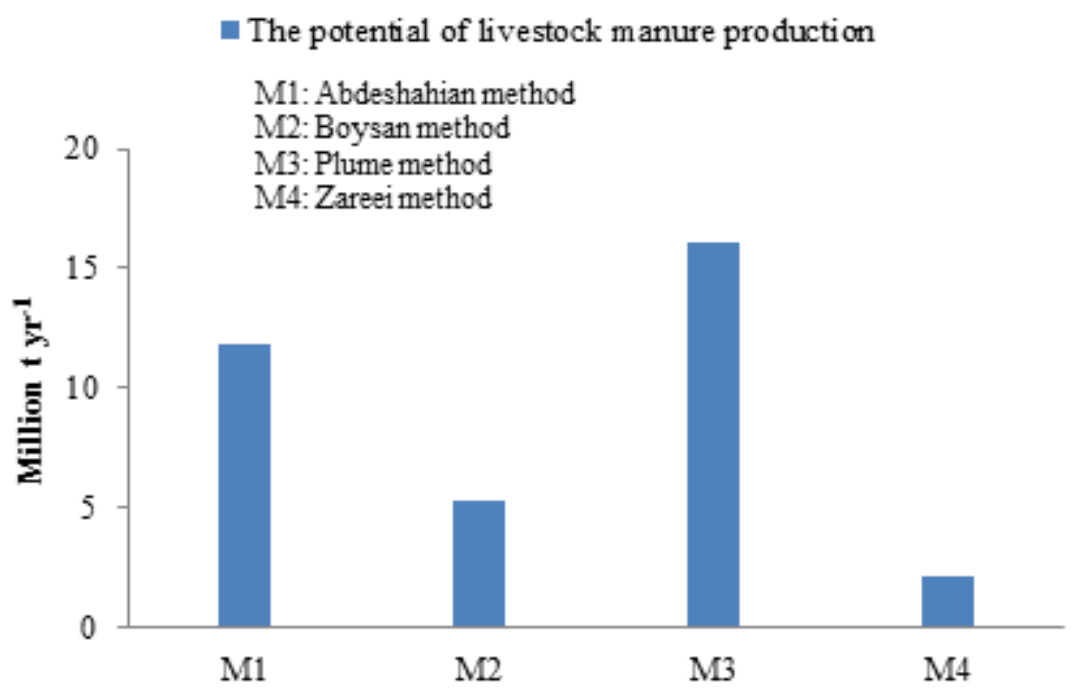

Figure 2. Comparison the estimated values of the manure production potential in Iran using 4 different calculation methods

The comparison of the biogas production potential in different provinces of Iran (Tables 8 and 10) revealed that Tehran had the highest potential of biogas evolution based on Abdeshahian, Zareei and Boysan methods, while Isfahan had the highest potential of biogas evolution based on Plume method. Hormozgan had the lowest amount of biogas production potential in each method.

The biogas production potential from the livestock manure based on 4 mentioned methods is shown in Figure 3. It is found that the highest potential of biogas from the manure is obtained from Abdeshahian method with the biogas production potential of 886.57 million $\mathrm{m}^{3} \mathrm{yr}^{-1}$, followed by Plume, Zareei and Boysan methods with a biogas generation potential of $745.37,565.85$ and 173.36 million $\mathrm{m}^{3} \mathrm{yr}^{-1}$, respectively.

This study showed that the cattle manure can be considered as the good source for the generation of biogas in an AD process. Similar observations were reported by other studies. For instance, it has been reported that Vietnam has the potential of generation 


$$
-6517-
$$

of $16878 \mathrm{TJ} \mathrm{y}^{-1}$ biogas in 2014 from the cattle manure (Roubik et al., 2017). The study on the Nigeria biogas potential has revealed that Nigeria biogas potential from livestock manure represents a minimum of $1.62 \times 109 \mathrm{~m}^{3}$ of biogas per annum (Adeoti et al., 2014).

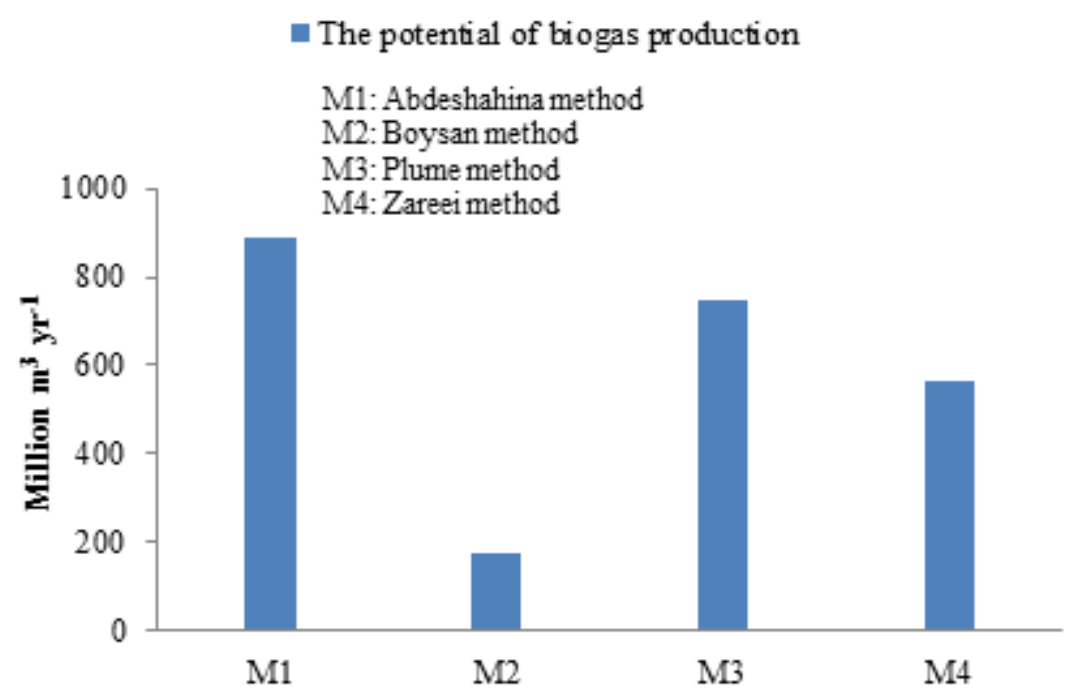

Figure 3. Comparison the estimated values of the biogas production potential in Iran using 4 different calculation methods

Figure 4 depicts the energy and electricity potential from livestock manure in Iran based on 4 calculation methods. As can be seen, the biogas produced from the livestock manure based on Abdeshahian, Boysan, Plume and Zareei methods was potentially able to provide the heat energy with the value of 5.14, 1, 4.32 and 3.28 thousand $\mathrm{GWh}_{\mathrm{yr}}{ }^{-1}$, respectively. The results shown in Figure 4 also indicated that the total amount of electricity generation estimated in Iran based on Abdeshahian, Boysan, Plume and Zareei methods was $1.59,0.31,1.34$ and 1.02 thousand $\mathrm{GWh} \mathrm{yr}^{-1}$, respectively. These estimated values could provide the $0.67 \%, 0.56 \%, 0.13 \%$ and $0.42 \%$ of the total electrical energy consumed in Iran in 2016 with the total electricity consumption of 237.4 billion kWh (Iran Energy Ministry, 2017). It should be noted that the electricity consumption with the value of 237.4 billion $\mathrm{kWh}$ was related to household, public, agricultural, industrial and other uses of the electrical energy consumed. The share of electrical energy consumption in household sector in Iran in 2016 was mainly for lightning, electrical household appliances and cooling systems with amount of $33 \%$ of the total electricity consumption. Hence, the electricity generation from livestock manure based on Abdeshahian, Plume, Boysan and Zareei methods could provide the $2.03 \%, 1.71 \%, 0.39 \%$ and $1.3 \%$ of the total electrical energy consumed in household sector in Iran in 2016, respectively, with the total electricity consumption of 78378 million $\mathrm{kWh}$.

In this regard, the investigation on the potential of electricity generation from livestock manure in Malaysia showed that the total amount of electricity generation was $6.85 \mathrm{E}+09 \mathrm{kWh} \mathrm{yr}^{-1}$ (Abdeshahian et al., 2016). Also the study on the potential for electricity generation from biogas in South Africa showed that the potential of electricity production from cattle manure was 2098553 MWh (Laks, 2017). 


$$
-6518-
$$

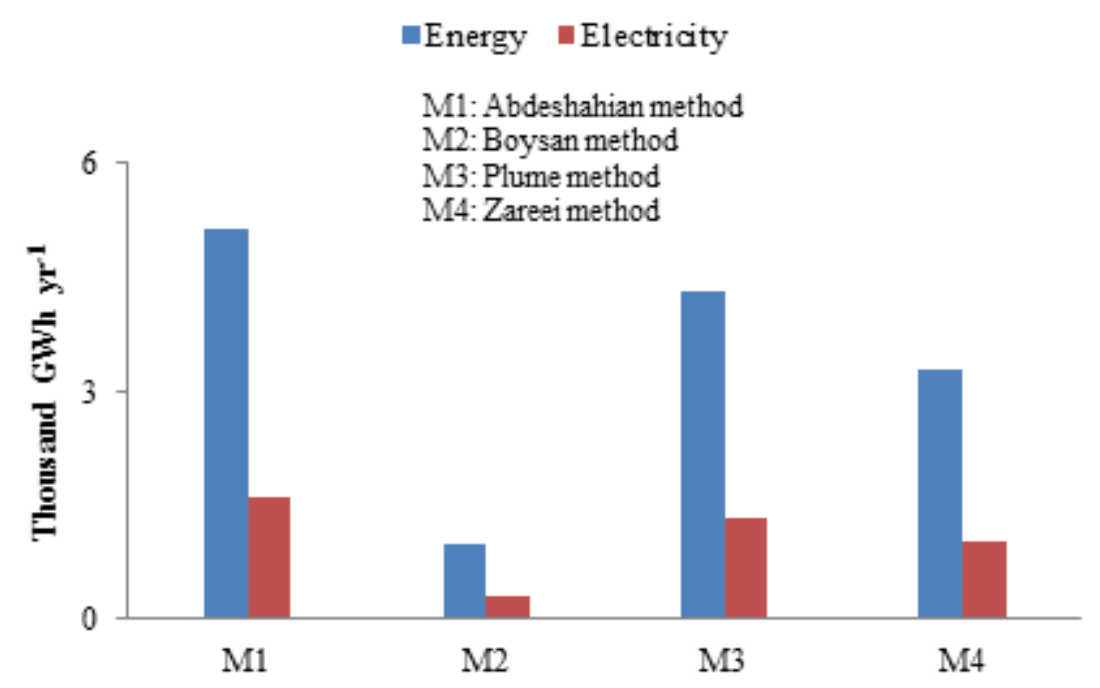

Figure 4. Comparison the estimated values of the energy and electricity potential from livestock manure in Iran using 4 different calculation methods

The methane production potential from the livestock manure in Iran with considering the different types of substrate (lipid, lignin, dry matter and cellulose) is shown in Figure 5. As can be observed, the highest potential of methane yield is related to the BMP value of lipid and lignin content at 5.27E $+10 \mathrm{NL} \mathrm{CH}_{4}$ digester $^{-1}$ day $^{-1}$ followed by BMP value of lipid, lignin and cellulose, BMP value of lipid, lignin and dry matter and BPM value of lipid, at, respectively, 5.25E + 10, 5.03E + 10 and 4.01E + $10 \mathrm{NL}$ $\mathrm{CH}_{4}$ digester ${ }^{-1}$ day $^{-1}$.

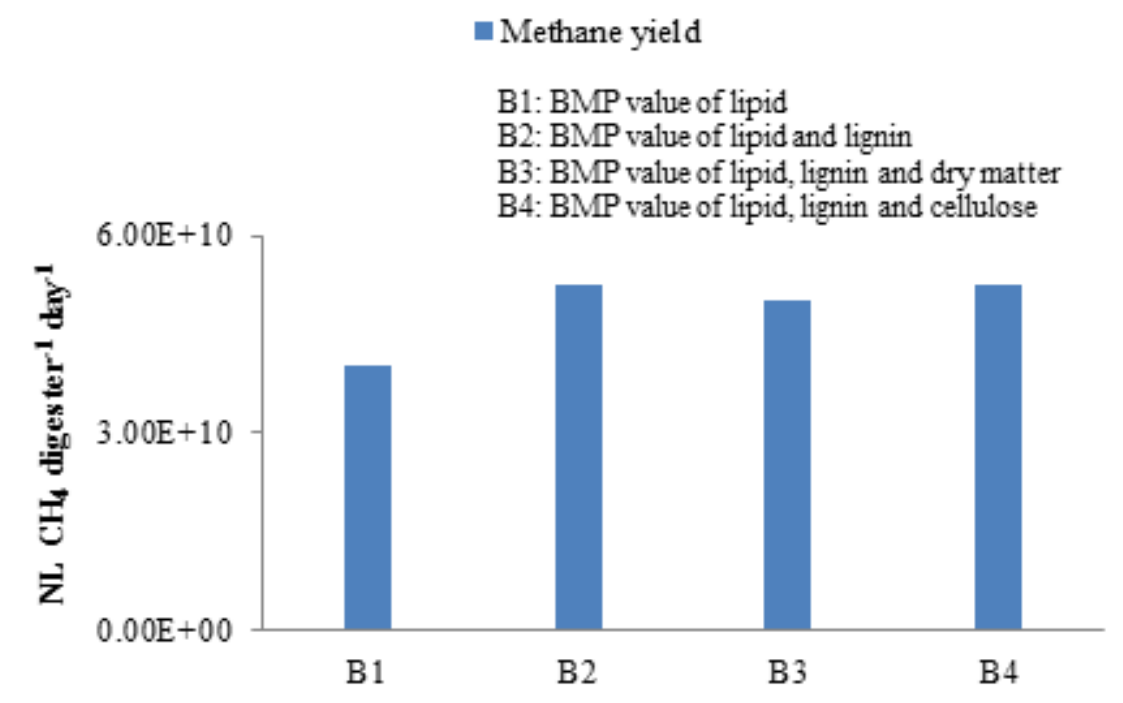

Figure 5. Comparison the estimated values of the methane production potential from livestock manure in Iran considering the BMP value of specific substrate 
Study fulfilled by Scarlat et al. (2018b) revealed that the potential of methane production from livestock manure in Belgium, Germany, Ireland, Spain and France is 556, 2907, 893, 2298, 3952 million $\mathrm{m}^{3}$, respectively. These results reflect the lower methane potential in mentioned countries than Iran. This discrepancy could be related to the variation in the biotransformation rate of manure into methane and different amount of the livestock waste produced.

\section{Conclusion}

Present study focuses on the evaluation of the different methods for estimating biogas production potential from livestock manure in Iran. Biogas generation from animal wastes has rarely been assessed based on their chemical structure and the findings of this research reveal the significance of manure chemical properties to be considered or the estimation of biogas production in husbandry industry. As a result of the calculations made, the manure production was estimated as 11.82, 5.25, 16.05 and 2.1 million ton $\mathrm{yr}^{-1}$ based on Abdeshahin, Boysan, Plume and Zareei methods with a biogas generation potential of $886.57,173.36,745.37$ and 565.85 million $\mathrm{m}^{3} \mathrm{yr}^{-1}$, energy equivalent of $5.14,1,4.32$ and 3.28 thousand $\mathrm{GWh} \mathrm{yr}^{-1}$, and electricity generation potential of $1.59,0.31,1.34$ and 1.02 thousand $\mathrm{GWh} \mathrm{yr}^{-1}$, respectively. The electrical energy values could provide the $2.03 \%, 1.71 \%, 0.39 \%$ and $1.3 \%$ of the total electrical energy consumed in household sector in Iran in 2016, respectively. Furthermore the methane yield potential from the livestock manure with considering the different types of substrate (lipid, lignin, dry matter and cellulose) was evaluated. The highest methane yield was obtained from BMP value based on lipid and lignin content at $5.27 \mathrm{E}+10 \mathrm{NL} \mathrm{CH}_{4}$ digester $^{-1}$ day $^{-1}$. This study shows that the treatment of the livestock manure by the anaerobic digestion process is helpful for producing the huge amounts of renewable energy as biogas. In addition, anaerobic digestion of livestock waste reduces their deleterious impacts on the environment and the treated organic matters could be used for the improvement of crops growth in the agriculture land. For future studies it is recommended to develop new applicable models based on chemical composition of animal wastes to further reveal the contribution of animal waste composition in generation of biochar and precisely estimate biogas production potential from such wastes.

\section{REFERENCES}

[1] Abdeshahian, P., Lim, J. S., Ho, W. S., Hashim, H., Lee. C. T. (2016): Potential of biogas production from farm animal waste in Malaysia. - Renewable and Sustainable Energy Reviews 60: 714-723.

[2] Achinas, S., Euverink, G. J. W. (2016): Theoretical analysis of biogas potential prediction from agricultural waste. - Resource-Efficient Technologies 2: 143-147.

[3] Adeoti, O., Ayelegun, T. A., Osho, S. O. (2014): Nigeria biogas potential from livestock manure and its estimated climate value. - Renewable and Sustainable Energy Reviews 37: 243-248.

[4] Afazeli, H., Jafari, A., Rafiee, S., Nosrati, M. (2014): An investigation of biogas production potential from livestock and slaughter house wastes. - Renewable and Sustainable Energy Reviews 34: 380-386. 
[5] Angelidaki, I., Treu, L., Tsapekos, P., Luo, G., Campanaro, S., Wenzel, H., Kougias, P. G. (2018): Biogas upgrading and utilization: Current status and perspectives. Biotechnology Advances 36: 452-466.

[6] Beldman, A., van Berkum, S., Kortstee, H., Zijlstra, J. (2017): Dairy farming and dairy industry in Iran. - Wageningen University \&Research.

[7] Benito, M., Ortiz, I., Rodriguez, L., Munoz, G. (2015): Ni-Co bimetallic catalyst for hydrogen production in sewage treatment plants: biogas reforming and tars removal. International Journal of Hydrogen Energy 40(42): 14456-14468.

[8] Boysan, F., Ozer, C, Bakkaloglu, K., Borekci, M. T. (2015): Biogas production from animal manure. - Procedia Earth and Planetary Science 15: 908-911.

[9] Burg, V., Bowman, G., Haubensak, M., Baier, U., Thees, O. (2018): Valorization of an untapped resource: Energy and greenhouse gas emissions benefits of converting manure to biogas through anaerobic digestion. - Resources, Conservation \& Recycling 136: 5362.

[10] Chandekar, A. C., Debnath, B. K. (2018): Computational investigation of air-biogas mixing device for different biogas substitutions and engine load variations. - Renewable Energy 127: 811-824.

[11] Chen, Y. R., Hashimoto, A. G. (1978): Kinetics of Methane Fermentation. Biotechnology and Bioengineering Symposium. - Wiley, New York, USA.

[12] Ch'ng, H. Y., Ahmed, O. H., Kassim, S., Majid, N. M. A. (2014): Recycling of sago (Metroxylon sagu) bagasse with chicken manure slurry through co-composting. - J. Agr. Sci. Tech. 16: 1441-1454.

[13] Comparetti, A., Greco, C., Navickas, K., Venslauskas, K. (2012): Evaluation of potential biogas production in Sicily. $-11^{\text {th }}$ International Scientific Conference: Engineering for Rural Development, Jelgava, Latvia.

[14] Costa, M. S. S. D. M., Jr, J. D. L., Costa, L. A. D. M., Orrico, A. C. A. (2016): A highly concentrated diet increases biogas production and the agronomic value of young bull's manure. - Waste Management 48: 521-527.

[15] Cu, T. T. T., Pham, H. C., Le, T. H., Nguyen, V. C., Le, X. A., Nguyen, X. T., Sven, G. C. (2012): Manure management practices on biogas and non-biogas pig farms in developing countries using livestock farms in Vietnam as an example. - Journal of Cleaner Production 27: 64-71.

[16] Cu, T. T. T., Nguyen, T. X., Triolo, J. M., Pedersen, L., Le, V. D., Le, P. D., Sommer. S. G. (2015): Biogas production from Vietnamese animal manure, plant residues and organic waste: influence of biomass composition on methane yield. - Asian Australas. J. Anim. Sci. 28(2): 280-289.

[17] De-Vries, J. W., Vinken, T. M. W. J., Hamelin, L., De-Boer, I. J. M. (2012): Comparing environmental consequences of anaerobic mono- and co-digestion of pig manure to produce bio-energy - a life cycle perspective. - Bioresource Technology 125: 239-248.

[18] Eze, J. I., Agbo, K. E. (2010): Studies on the microbial spectrum in an aerobic biomethanization of cow dung in $10 \mathrm{~m}^{3}$ fixed dome biogas digester. - International Journal of the Physical Sciences 5(8): 1331-1337.

[19] Garcia, A. P. (2014): Techno-economic feasibility study of a small-scale biogas plant for treating market waste in the city of El Alto. - Master of Science Thesis, KTH School of Industrial Engineering and Management, Division of Energy and Climate, Stockholm.

[20] Gebrezgabher, S. A., Meuwissen, M. P. M., Prins, B. A. M., Lansink, A. G. J. M. O. (2010): Economic analysis of anaerobic digestion - a case of Green power biogas plant in The Netherlands. - NJAS - Wageningen Journal of Life Sciences 57(2): 109-115.

[21] Government of Islamic Republic of Iran (GOI)/Ministry of Jihad-e-Agriculture (MOJA), Food and Agriculture Organization (FAO) of the United Nations (2012): Country Programming Framework (CPF) 2012-2016 for Iran's Agriculture Sector. - GOI, MOJA, FAO, Tehran, Rome. 
[22] Hamzeh, Y., Ashori, A., Mirzaei, B., Abdulkhani, A., Molaei, M. (2011): Current and potential capabilities of biomass for green energy in Iran. - Renewable and Sustainable Energy Reviews 15: 4934-4938.

[23] Hashimoto, A. G., Chen, Y. R., Varel, V. H. (1981): Theoretical aspects of methane production: State-of-the-art. - Livestock Wastes: A Renewable Resource - $4^{\text {th }}$ International Symposium on Livestock Wastes, ASAE, pp. 86-91.

[24] Holm-Nielsen, J. B., Seadi, T. A., Oleskowicz-Popiel, P. (2009): The future of anaerobic digestion and biogas utilization. - Bioresource Technology 100(22): 5478-4584.

[25] Hosseini, S. E., Wahid, M. A. (2014): Development of biogas combustion in combined heat and power generation. - Renewable and Sustainable Energy Reviews 40: 868-875.

[26] Ilaboya, I. R., Asekhame, F. F., Ezugwu, M. O., Erameh, A. A., Omofuma, F. E. (2010): Studies on biogas generation from agricultural waste; analysis of the effects of alkaline on gas generation. - World Appl. Sci. J. 9(5): 537-545.

[27] International Energy Agency (2015): Energy and Climate Change. - World Energy Outlook Special Report, OECD/IEA, Paris.

[28] Iran Energy Ministry (2017): Iran Power Industry 2016. - Tavanir Holding Company, Iran (Persian).

[29] Kougias, P. G., Boe, K., Angelidaki, I. (2013): Effect of organic loading rate and feedstock composition on foaming in manure-based biogas reactors. - Bioresource Technology 144: 1-7.

[30] Laks, R (2017): The Potential for Electricity Generation from Biogas in South Africa. ECN-E--17-001. ECN, Petten, Netherlands

[31] Luostarinen, S. (2013): Energy Potential of Manure in the Baltic Sea Region: Biogas Potential \& Incentives and Barriers for Implementation. - Knowledge Report, Baltic Forum for Innovative Technologies for Sustainable Manure Management.

[32] Mata-alvarez, J., Mace, S., Llabres, P. (2000): Anaerobic digestion of organic solid wastes - an overview of research achievements and perspectives. - Bioresource Technology 74(1): 3-16.

[33] Mathias, J. F. C. M. (2014): Manure as a resource: livestock waste management from anaerobic digestion, opportunities and challenges for Brazil. - International Food and Agribusiness Management Review 17: 87-110.

[34] Meyer, A. K. P., Ehimern. E. A., Holm-Nielsen, J. B. (2018): Future European biogas: Animal manure, straw and grass potentials for a sustainable European biogas production. - Biomass and Bioenergy 111: 154-164.

[35] Mohammadi Maghanaki, M., Ghobadian, B., Najaf, G., Janzadeh Galogah, R. (2013): Potential of biogas production in Iran. - Renewable and Sustainable Energy Reviews 28: 702-714.

[36] Nasir, I. M., Ghazi, T. I. M., Omar, R. (2012): Anaerobic digestion technology in livestock manure treatment for biogas production: a review. - Engineering in Life Sciences 12: 258-269.

[37] Nasir, I. M., Ghazi, T. I. M., Omar, R., Idris, A. (2013): Anaerobic digestion of cattle manure: influence of inoculums concentration. - International Journal of Engineering Technology 10(1): 22-26.

[38] Neshat, S. A., Mohammadi, M., Najafpour, G. D., Lahijani, P. (2017): Anaerobic codigestion of animal manures and lignocellulosic residues as a potent approach for sustainable biogas production. - Renewable and Sustainable Energy Reviews 79: 308322.

[39] Omar, R., Harun, R. M., Ghazi, T. I. M., Wan-Azlina, W. A. K. G., Idris, A., Yunus, R. (2009): Anaerobic treatment of cattle manure for biogas production. - Proceedings of the annual meeting of American Institute of Chemical Engineers, Philadelphia, USA.

[40] Omrani, GH. (1996): Basics Biogas Production from Urban and Rural Waste. University of Tehran Publication, Iran. 
[41] Onurbas-Avcioglu, A., Turker, U. (2012): Status and potential of biogas energy from animal wastes in Turkey. - Renewable Sustainable Energy Reviews 16(3): 1557-1561.

[42] Ounnar, A., Benhabyles, L., Igoud, S. (2012): Energetic valorization of biomethane produced from cow-dung. - Procedia Engineering 33: 330-334.

[43] Ozer, B. (2017): Biogas energy opportunity of Ardahan city of Turkey. - Energy 139: 1144-1152.

[44] Perrigault, T., Weatherford, V., Marti-Herrero, J., Poggio, D. (2012): Towards thermal design optimization of tubular digesters in cold climates: A heat transfer model. Bioresource Technology 124: 259-268.

[45] Pessuto, J., Scopel, B. S., Perondi, D., Godinho, M., Dettmer, A. (2016): Enhancement of biogas and methane production by anaerobic digestion of swine manure with addition of microorganisms isolated from sewage sludge. - Process Safety and Environmental Protection 104: 233-239.

[46] Plume, I., Dubrovskis, V., Plume, B. (2012): Specified evaluation of manure resources for production of biogas in planning region Latgale. - International Scientific Conference of Renewable Energy and Energy Efficiency, Latvia University of Agriculture.

[47] Rennuit, C., Sommer, S. G. (2013): Decision support for the construction of farm-scale biogas digesters in developing countries with cold seasons. - Energies 6(10): 5314-5332.

[48] Roubik, H., Mazancova, J., Phung, L. D., Dung. D. V. (2017): Quantification of biogas potential from livestock waste in Vietnam. - Agronomy Research 15: 540-552.

[49] Sahota, S., Shah, G., Ghosh, P., Kapoor, R., Sengupta, S., Singh, P., Vijay, V., Sahay, A., Vijay, V. K., Thakur, I. S. (2018): Review of trends in biogas upgradation technologies and future perspectives. - Bioresource Technology Reports 1: 79-88.

[50] Samun, I., Saeed, R., Abbas, M., Rehan, M., Nimazi, A. S., Asam, Z. U. Z. (2017): Assessment of bioenergy production from solid waste. - Energy Procedia 142: 655-660.

[51] Santos, R. O. D., Santos, L. D. S., Prata, D. M. (2018): Simulation and optimization of a methanol synthesis process from different biogas sources. - Journal of Cleaner Production 186: 821-830.

[52] Scarlat, N., Dallemand, J-F., Fahl, F. (2018): Biogas: developments and perspectives in Europe. - Renewable Energy. DOI: 10.1016/ j.renene.2018.03.006.

[53] Scarlet, N., Fahl, F., Dallemand, J. F., Monforti, F., Motola, V. (2018): A spatial analysis of biogas potential from manure in Europe. - Renewable and Sustainable Energy Reviews 94: 915-930.

[54] Statistical Centre of Iran (2017): Statistical Surveys of Dairy Industry in Iran, 2016. Statistical Centre of Iran. Office of the Head, Public Relations and International Cooperation, Tehran (Persian).

[55] Tehran Chamber of Commerce, Industries, Mines and Agriculture, Deputy of Economic Research (2016): Raw Milk Production from Industrial Cattle Farms in Tehran in 2016. Tehran Chamber of Commerce, Tehran (Persian).

[56] Than, T. M. M. (2005): Myanmar's energy sector: banking on natural gas. - Southeast Asian Affairs 257-289.

[57] Travnicek, P., Kotek, L., Junga, P., Vítez, T., Drapela, K., Chovanec, J. (2018): Quantitative analyses of biogas plant accidents in Europe. - Renewable Energy 122: 8997.

[58] United Nations Environment Programme (2014): The Emissions Gap Report. - A UNEP Synthesis Report, UNEP, Nairobi.

[59] Wang, Y., Wu, X., Tong, X., Li, T., Wu, F. (2018): Life cycle assessment of large-scale and household biogas plants in northwest China. - Journal of Cleaner Production 192: 221-235.

[60] Wyman, C. E., Goodman, B. J. (1993): Biotechnology for production of fuels, chemicals and materials from biomass. - Applied Biochemistry and Biotechnology 39: 41-59. 


$$
-6523-
$$

[61] Yazan, D. M., Fraccascia, L., Mes, M., Zijm, H. (2018): Cooperation in manure-based biogas production networks: An agent-based modeling approach. - Applied Energy 212: 820-833.

[62] Yildirim, E., Ince, O., Aydin, S., Ince, B. (2017): Improvement of biogas potential of anaerobic digesters using rumen fungi. - Renewable Energy 109: 346-353.

[63] Zareei, S. (2018): Evaluation of biogas potential from livestock manures and rural wastes using GIS in Iran. - Renewable Energy 118: 351-356. 